Electrical and mechanical field interactions of piezoelectric systems: foundation of smart structures-based piezoelectric sensors and actuators, and free-fall sensors

This article has been downloaded from IOPscience. Please scroll down to see the full text article.

2004 Smart Mater. Struct. 131090

(http://iopscience.iop.org/0964-1726/13/5/015)

The Table of Contents and more related content is available

Download details:

IP Address: 140.112.113.225

The article was downloaded on 25/12/2008 at 02:27

Please note that terms and conditions apply. 


\title{
Electrical and mechanical field interactions of piezoelectric systems: foundation of smart structures-based piezoelectric sensors and actuators, and free-fall sensors*
}

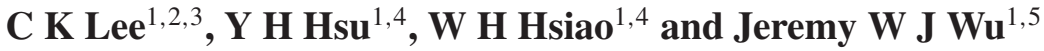 \\ ${ }^{1}$ Institute of Applied Mechanics, National Taiwan University, No. 1, Section 4, Roosevelt \\ Road, Taipei, Taiwan \\ ${ }^{2}$ Department of Engineering Science and Ocean Engineering, National Taiwan University, \\ Taipei, Taiwan
}

Received 22 April 2003, in final form 1 June 2004

Published 6 August 2004

Online at stacks.iop.org/SMS/13/1090

doi:10.1088/0964-1726/13/5/015

\begin{abstract}
Based on an idea to fully integrate the advantages of both distributed and point sensors, we adopted mathematical tools such as method of image, linear superposition, and window functions to develop a fundamental approach towards creating no-phase delay filters for finite sensor structures. Through an expanded design freedom for piezoelectric sensors and actuators as a result of adding in situ, we can obtain a no-phase delay signal filtering capability by properly designing an effective surface electrode onto the piezoelectric layer. An inertia-based free-fall sensor that can measure the start of free-fall motion in addition to creating a piezoelectric sensor and actuator pair that possesses a spatial dependent transfer function and demonstrates the wide range applicability of the new concepts are disclosed. The fundamental perspective of pursuing signal processing through an electromechanical interaction of wave modes and of piezoelectric materials is examined in detail. It can be shown that the fundamental design criteria of electromechanical-coupled systems are highly related to the temporal and spatial characteristics of the mechanical structure and its electrical interaction between interface circuits. After pursuing the physical characteristics of these systems, the possibilities of extending the no-phase delay signal processing capabilities to other physical fields are discussed as well.
\end{abstract}

\section{Introduction}

Direct and converse piezoelectric effects have served as excellent platforms to link mechanical and electrical fields

\footnotetext{
* Partial contents of this paper were presented as the Plenary Speech at the SPIE 10th Annual International Symposium on Smart Structures and Materials under the same title.

3 Author to whom any correspondence should be addressed.

4 Present address: Advance Wave Technology, Inc., Suite A, 10 Floor, No 56, Section 4, Nanjing East Road, Taipei, Taiwan.

5 Present address: Department of Engineering Science and Ocean Engineering, National Taiwan University, Taipei, Taiwan.
}

over the years [1]. Many applications based on exploiting the piezoelectric effect have been developed, including those of crystal oscillators, SAW devices, piezoelectric inkjet printer heads, and piezoelectric accelerometers. All these piezoelectric systems have incorporated different piezoelectric operating methods, different mechanical structures, and different frequency ranges, many of which have varied highly with respect to the application. In this paper, a causal signal processing effect that appears to circumvent the Bode gainphase theorem [2] will be detailed. The Bode theorem basically states that if gain tailoring is applied to a transfer 


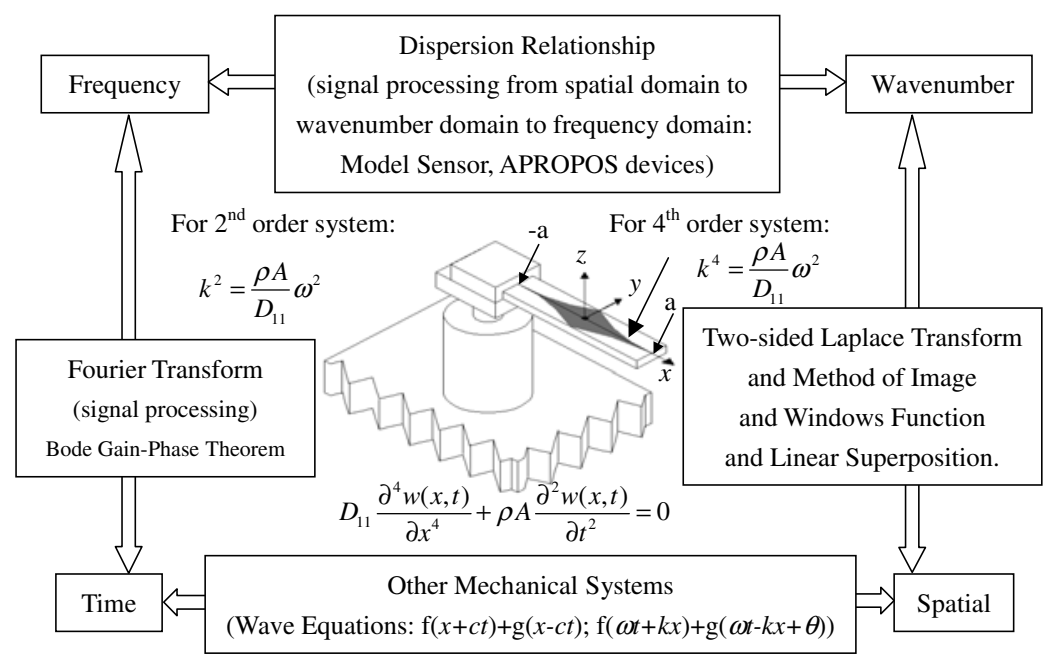

Figure 1. Transformation/signal processing in a mechanical system.

function of a minimum phase system, a phase delay is not only inevitable but is also uniquely determined by the gain tailoring function. Since its inception, this fundamental understanding has governed all work on signal processing and control efforts. A signal filtering that can tailor a gain transfer function to a sensor system without suffering from related phase delay consequences as predicted by Bode gainphase theorem will be shown to be feasible both theoretically and experimentally. To properly identify the above-mentioned autonomous/independent gain-phase tailoring properly and to clearly state that piezoelectric systems can serve well as one of the fundamental platforms to execute this new-found signal processing freedom, an acronym APROPOS (autonomous phase-gain rotational/linear piezoelectric optimal sensing) was coined to facilitate the new concept discussed in this paper. Detailed findings of these APROPOS devices were previously reported by $\mathrm{Hsu}$ and Lee [3, 4]. It was shown that APROPOS devices derive their associated capabilities by properly utilizing transformations among all fields of mechanical systems, which includes time, frequency, spatial, and wavenumber domains (figure 1). A brief review of this new innovative device will be introduced to import the developed fundamental design criteria of electromechanicalcoupled systems.

Metrology techniques that can be used to evaluate or improve the performance of flexible structures have been actively developed since the 1980s [5]. Both piezoelectric point sensors and distributed sensors (figure 2) have been widely implemented to sense structure performance. In addition, both are the two most representative electromechanical-coupled systems over the last 20 years. The advantage of a point sensor, which includes the majority of today's sensors such as accelerometers, strain gages, etc, is that the user does not need to know the full details of the sensor design to detect the vibration of arbitrary structures directly. However, it is known that the usable bandwidth of a point sensor is typically $1 / 10-1 / 5$ of its first resonance frequency if linearity and accuracy are determined to be better than $5-10 \%$ [6]. This is because the performance of a point sensor is tightly related to the sensor structure that is typically determined by the resonant characteristics such as gain and phase of the chosen sensor structure.

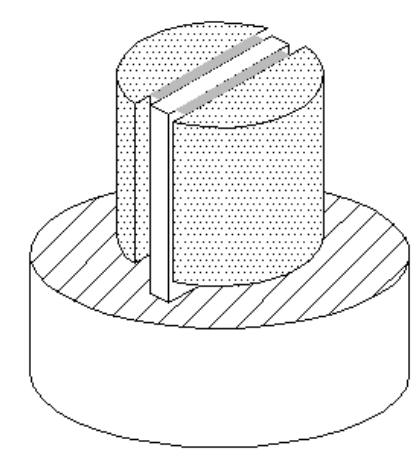

(a)

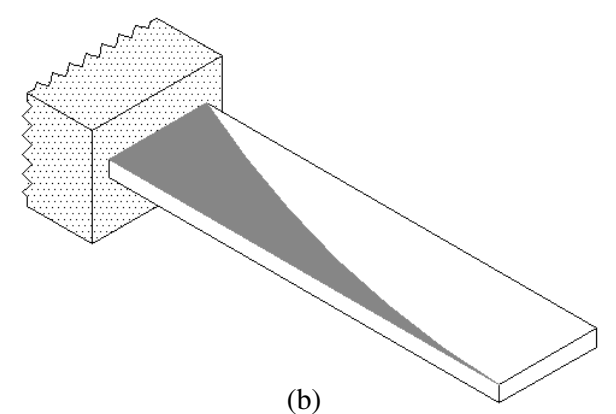

Figure 2. Types of piezoelectric sensors: (a) point sensors and (b) distributed sensors

In other words, the performance of a point sensor is determined at the time the sensor structure is chosen. On the other hand, the concept of using a distributed sensor to control a flexible structure has been studied widely for the last 20 years [5, 79]. Many innovative piezoelectric distributed sensors such as modal sensors [8] and spatial filters [9] were thus developed which tailor the layout of piezoelectric materials on the sensor structure to influence sensor transfer functions. For example, a modal sensor uses a shaped piezoelectric lamina to match one of the strain mode shapes of a finite flexible structure and applies the orthogonal property of finite structures to reject the influence of other resonance modes [8]. The matched resonance mode is thus the only structural information detected 
by the modal sensor. In comparison, a spatial filter offers a weighting function in a spatial domain with a shaped distributed sensor by assuming the flexible structure of interest is infinite in length [9]. Thus a no-phase delay low-pass filter is created that appears to violate the Bode gain-phase theorem but actually proves to be valid for electromechanical systems.

Despite the long-term research on distributed piezoelectric sensors and actuators, they remain less popular when compared to point sensors. This shortcoming can be attributed to the fact that these innovative distributed sensors must be redesigned for each structure. Nevertheless, it is worth noting that the distributed sensor research results point out a series of innovative thoughts, i.e., the spatial characteristics of mechanical structures can reveal valuable vibration information. Armed with the understanding from previously developed distributed sensors, attempts to fully integrate the advantages of both worlds led us to the development of a new class of point sensors called point-distributed sensors ( $P o D$ sensors for short) [3]. It should be noted that the reciprocal relationship of piezoelectric materials provides us with an opportunity to extend all discussions on $P o D$ sensors to pointdistributed actuators ( $P o D$ actuators). The combination of $P o D$ sensors and $P o D$ actuators opens up the possibility of developing an active type of $P o D$ sensor by following the same path as traditional active sensors $[3,10]$. Once the theoretical foundation of the above-mentioned APROPOS device and $P o D$ sensors were developed, a series of piezoelectric sensors include a free-fall sensor that can measure the start of free-fall motion [11] was used to experimentally demonstrate the range of applicability for these newly developed ideas.

It can be shown that the transformation exists within many mechanical systems (figure 1) that derive their origins from their governing equations that are actual partial differential equations in nature. That is, structure vibrations are a function of time and space. By fully exploring the spatial and wavenumber domain behavior embedded within partial differential equations, the effect of the interaction between the actuator and sensor pair on structure control was further studied to understand the potential impact revealed by the design criteria proposed herein. The pole-zero alternating phenomenon between the pointed sensor and actuator pair on a flexible structure that has the advantage of increasing the phase margin in a control loop was first reported and applied by Martin at 1978 [12]. Martin stated that the zeros in the transfer function between the sensor and actuator are the results of the locations chosen by the devices on the flexible structure. Martin also proposed the concept of a collocated sensor and actuator pair that ensures the poles and zeros alternate in the transfer function. It is to be noted that Martin does demand 'collocated' to represent both the same location and reciprocal sensor and actuator pairs. The study revealed that the spatial relationship between sensor and actuator pairs will influence the characteristic of the transfer function.

It will be further clarified that when considering general distributed piezoelectric sensors and actuators, the pole-zero alternating phenomenon can be controlled by both the location and the sensor and actuator surface electrode shapes. More specifically, the system transfer function of this type of system is actually governed by the location and the surface electrode shapes of the sensors and actuators involved. Based on our theoretical and experimental work, we can show that since the shape and location of both the point and distributed sensor/actuator contribute to the system transfer function, applying spatial information to pursue unconventional sensor and actuator signal processing within piezoelectric systems is not only feasible but also leads to effects not known to be possible before. Based on the approach depicted in this paper, we can show that both spatial characteristics and temporal information must be incorporated to take full advantage of the design of electromechanical-coupled systems.

\section{Design concept of point-distributed sensors}

As mentioned above, today's point sensors have their performance parameters set at the time the sensor structure is determined. In other words, these sensors cannot break free from the generic limitations of the sensor structure on which they are based. This issue is of particular importance with the recent rapid development of micromachining-based silicon accelerometers [13] called MEMS (micro-electromechanical systems) accelerometers. These sensors are typically very small and the sensor structure created by using the silicon etching process tends to be more limited in terms of design freedom when compared to traditional accelerometers. To make sure that MEMS accelerometers, and certainly all associated MEMS devices included, have just as wide operational space, a new design of freedom was attempted at the beginning of this research. The thought was to bring the transfer function tailoring capabilities found in distributed piezoelectric sensors into the design toolbox of piezoelectric point sensors. The configuration of a typical $P o D$ sensor can be seen in figure 1 . This configuration treats the sensor structure like a one-dimensional cantilever plate as a flexible structure, where the sensor implemented is a distributed type from a sensor structure perspective. However, the overall sensor developed is used as a point sensor. This design approach brings the benefits of a distributed sensor into a point sensor design and thus clearly expands the horizon of all piezoelectric sensor design approaches.

Typically there are two approaches to raise the lowfrequency sensitivity of a point sensor. The first approach is to make the sensor structure more flexible and thus more sensitive in a low-frequency range, which inevitably lowers the first resonant frequency of the system. The second approach is to increase the gain of the amplifiers used to amplify the sensor signal. Both approaches suffer from the fact that when noise becomes amplified by the resonant behaviors of the sensor structure, it may saturate the whole interface circuitry. In addition, both approaches must make compromises on the acceptable phase delay associated with the above-mentioned approach. A naïve approach will be to develop a gain tailoring no-phase delay filter, which is forbidden by the Bode gainphase theorem (figure 3). In figure 3, $G_{\mathrm{t}}(s)$ (thin dark curve) represents the transfer function of a traditional point sensor with a simulated transfer function $1 /\left(s^{2}+0.1 s+9\right)$, and $G_{\mathrm{o}}(s)$ (thick gray curve) represents the transfer function of a flexible structure (figure 1) with a simulated transfer function $1 /\left(s^{2}+0.4 s+1\right)$. Note that the transfer function of the point sensor $G_{\mathrm{t}}(s)$ was chosen to be three times higher than $G_{\mathrm{o}}(s)$ to simulate the situation of a traditional point sensor which often 


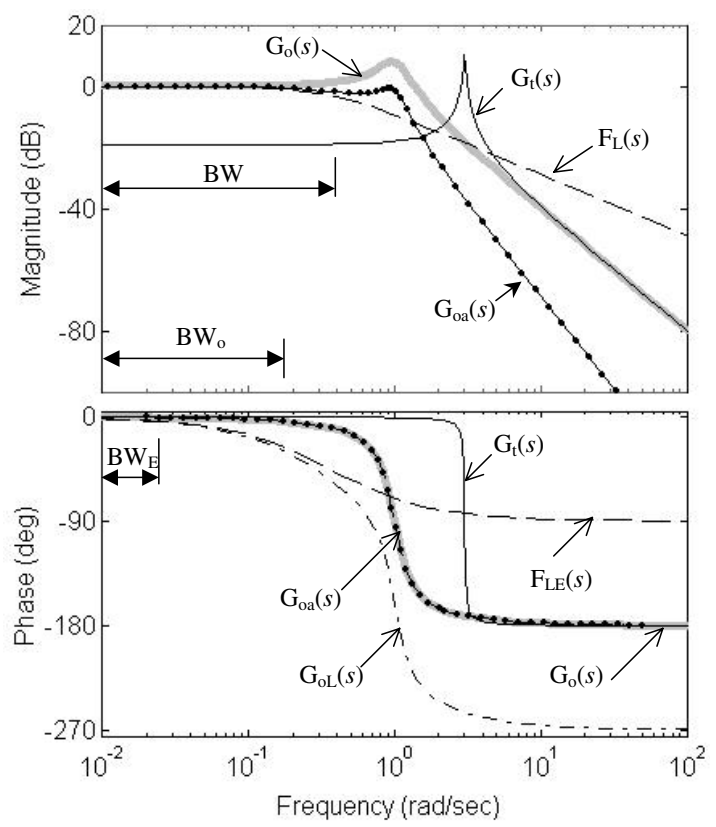

Figure 3. Effect of a no-phase delay filtering on sensor transfer function as shown by using a Bode plot.

has a high resonance frequency to have a usable bandwidth BW. On the other hand, the flexible sensor structure $G_{0}(s)$ offers a low enough frequency sensitivity, but has a limited usable bandwidth $\mathrm{BW}_{\mathrm{o}}$. The fundamental concept of the $P o D$ sensor is to introduce a kind of no-phase delay low-pass filter $F_{\mathrm{L}}(s)$ (dashed curve) like the APROPOS device proposed, into the sensor transfer function $G_{\mathrm{o}}(s)$ to reduce the effect of structure resonance and to increase the usable bandwidth from $\mathrm{BW}_{\mathrm{o}}$ back to $\mathrm{BW}$, shown as $G_{\mathrm{oa}}(s)$ (thin dark curve with dots). It should be noted that the usable bandwidth can be extended to BW only if the newly introduced low-pass filter does not induce any additional phase delay to the original phase function. This no-phase delay requirement cannot be offered by traditional electrical filters as phase lag like $F_{\mathrm{LE}}(s)$ (dashed curve) in figure 3 with respect to its corner frequency will inevitably be introduced which will automatically reduce the usable bandwidth to a much shorter $\mathrm{BW}_{\mathrm{E}}$ (see $G_{\mathrm{oL}}(s)$ (dashdotted curve)). More specifically, the $P o D$ sensor presented herein acts as a point sensor while having the advantage of possessing a good low-frequency sensitivity in addition to a wide usable bandwidth derived from the distributed sensor technology.

Two types of distributed sensor development provide us with hints on how to circumvent the limitation imposed by that of the Bode gain-phase theorem. The first is based on modal sensors developed by Lee and Moon in 1990 that possess the capability to measure a specific modal coordinate of a vibrating structure [8]. The second potential approach is based on introducing spatial filters, i.e., performing signal processing through a spatial domain signal integration/process, as discussed by Miller et al in 1990 [9]. Although modal sensors are applicable to finite structures, the spatial filter can only be adopted to infinite structures, rigorously speaking. The first attempt using spatial filters was undertaken by implementing a $7 \mathrm{~cm}$ long piezoelectric lamina placed far from the boundaries of a $730 \mathrm{~cm}$ long free-free onedimensional plate, which was assumed to be infinite in length. By assuming the test structure as being infinite, contributions of the evanescent waves and boundaries were then eliminated, which simplified the sensor equation into a two-sided Laplace transform [9, 14]. By introducing proper weighting functions in the spatial domain and then through the two-sided Laplace transform, a no-phase delay low-pass filter was proven possible [9]. The omission of the evanescent waves and the boundary effects are thus the limiting factors in using spatial filters within the realm of developing $P o D$ sensors. More specifically, spatial filters cannot function near or on the boundaries under conditions with low wavenumber (frequency) ranges or in small testing structures, i.e., they cannot be used to design $P o D$ sensors that have flexible sensor structures comparable in size to the piezoelectric materials adopted. It can be shown herein that the APROPOS device breaks free of this limitation by introducing either the mathematical concept of a windows function [15] or a method of image [16].

\subsection{Concept of an APROPOS device}

The fundamental design concept of the APROPOS device reported by Hsu and Lee in 2002 [3] is briefly detailed. The sensor equation of a one-dimensional plate (figure 1) derived from the piezoelectric lamina theory is $[8,17]$

$$
q_{k}(t)=-z_{k} e_{31} \int_{-a}^{a} R(x) \frac{\partial^{2} w}{\partial x^{2}} \mathrm{~d} x,
$$

where $z_{k}$ is the $k$ th layer of the piezoelectric lamina, $e_{31}$ is the piezoelectric stress/charge constant, $2 a$ is the length of the piezoelectric lamina along the $x$-axis, and $w$ is the bending displacement of the one-dimensional plate. The $R(x)$ is the effective surface electrode of

$$
R(x)=\int_{-b / 2}^{b / 2} F(x, y) P_{0}(x, y) \mathrm{d} y,
$$

where $b$ is the width of the piezoelectric lamina, and $F(x, y)$ and $P_{0}(x, y)$ are the weighting function and the poling strength of the effective surface electrode in the spatial domain, respectively. In this paper, the poling strength throughout the piezoelectric lamina will be considered to have a value of one, i.e., uniform poling. During the last two decades, the effective surface electrode $R(x)$ has been chosen as the foundation of many innovative distributed sensors. For example, a modal sensor [8] or a spatial filter [9] can be implemented by setting the effective surface electrode equal to some proper mathematical function as mentioned above. It was shown that an APROPOS device was derived from a similar mathematical formulation [3].

The governing equation of a one dimensional cantilever plate formed by laminating a piece of $52 \mu \mathrm{m}$ thick piezoelectric polymer lamina made of $\mathrm{PVF}_{2}$ [18] on top of a $200 \mu \mathrm{m}$ thick stainless steel shim (figure 1) where the internal damping effect is assumed to be negligible, can be expressed as follows $[8,9,17]$ :

$$
D_{11} \frac{\partial^{4} w(x, t)}{\partial x^{4}}+\rho A \frac{\partial^{2} w(x, t)}{\partial t^{2}}=0,
$$




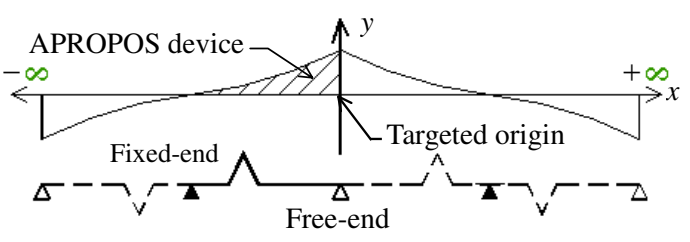

(a)

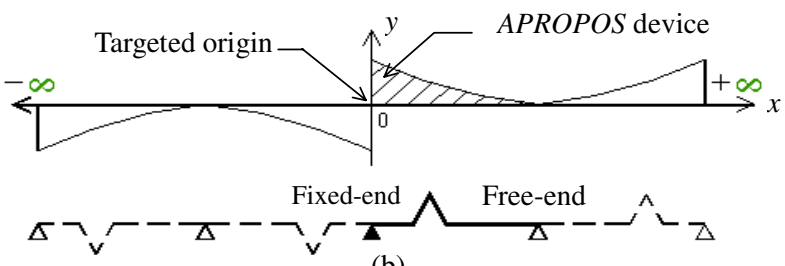

(b)

Figure 4. Schematic of (a) an even-function and (b) an odd-function APROPOS device, both of which have targeted origins set at a free end and a fixed end, respectively.

$$
k^{4}=\frac{\rho A}{D_{11}} \omega^{2},
$$

where $D_{11}, \rho$, and $A$ represent the flexural rigidity, density, and cross-section of the plate, respectively. Equation $(3 b)$ shows the dispersion relationship between wavenumber $k$ and frequency $\omega$. As the mechanical stiffness of the $\mathrm{PVF}_{2}$ thin lamina is much smaller than that of a stainless steel shim plate, its mechanical stiffness can be neglected. The general solution for equation ( $3 a$ ) can be expressed as [9]

$$
w(x, t)=\left[w_{\text {lp }} \mathrm{e}^{\mathrm{j} k x}+w_{\text {le }} \mathrm{e}^{k x}+w_{\text {rp }} \mathrm{e}^{-\mathrm{j} k x}+w_{\text {re }} \mathrm{e}^{-k x}\right] \mathrm{e}^{\mathrm{j} \omega t},
$$

where $\mathrm{j}=\sqrt{-1}, w_{\text {lp }}$ and $w_{\text {rp }}$ are the wave mode amplitudes of the left and right propagating wave modes, and $w_{\text {le }}$ and $w_{\text {re }}$ are the wave mode amplitudes of the left and right evanescent wave modes, respectively. These four wave mode amplitudes are determined by the boundary conditions of the one-dimensional cantilever plate. The wave mode amplitudes will be different for different structures, which, along with the wave modes, represent the characteristics of the various structures. Substituting equation (4) into (1) leads to the sensor equation

$$
\begin{aligned}
q(t) & =-z_{k} e_{31} \mathrm{e}^{\mathrm{j} \omega t} k^{2} \int_{-a}^{a} R(x)\left[-\left(w_{\mathrm{lp}} \mathrm{e}^{\mathrm{j} k x}+w_{\mathrm{rp}} \mathrm{e}^{-\mathrm{j} k x}\right)\right. \\
& \left.+\left(w_{\mathrm{le}} \mathrm{e}^{k x}+w_{\mathrm{re}} \mathrm{e}^{-k x}\right)\right] \mathrm{d} x .
\end{aligned}
$$

To eliminate the contributions of the evanescent waves, the spatial filter concept [9] developed in 1990 was placed far away from the boundaries. In this way the propagating wave modes $\mathrm{e}^{\mathrm{j} k x}$ and $\mathrm{e}^{-\mathrm{j} k x}$ represent the two-sided Laplace transform parameter and $R(x)$ the spatial function to be transformed to wavenumber domain $k$.

We can see that the only hindrance of treating equation (5) as a two-sided Laplace transform [3, 14] for conversion between the spatial and the wavenumber domains lies in the fact that the sensor structure of interest is finite and that we can mathematically map the finite structure into an infinite structure. The two methodologies adopted by the APROPOS device are the method of image $[3,16]$ (figure 4) and window functions [15]. As the method of image requires an integrant which corresponds to the product of the wave modes and where the effective surface electrode $R(x)$ in equation (5) be continuous throughout the expanded domain, a proper $R(x)$ must be chosen to facilitate the introduction of the method of image. The concepts of utilizing the method of image to expand the APROPOS device into an infinite domain have been detailed thoroughly by Hsu and Lee [3, 4]. With the introduction of a window function, we can detail a more powerful design tool for APROPOS devices.

A one-dimensional cantilever plate shown in figure 4 was chosen as an example since a fixed end and a free end are two of the most popular boundary conditions. It should be noted that the gain and phase of the wave modes depend highly on the characteristics of the boundaries, whose effects must be properly taken care of when expanding all modes into an infinite domain. Taking the fixed end as an example (figure 4), the reflected wave has a $180^{\circ}$ phase change with respect to the original once it hits the fixed end, i.e., the wave mode becomes discontinuous at the fixed end. More specifically, $R(x)$ must be zero at this type of boundary condition. In comparison, no amplitude or phase change with respect to the free end when the wave modes are reflected from it, simplifies the choice for $R(x)$. Examining equation (5) by using the method of image leads to a straightforward observation that the choice of $R(x)$ depends highly on the location of the coordinate origin. Furthermore, the sensor output $q(k)$ shown in equation (5) depends on the integrant that represents the product of the strain measured at the origin and the effective surface electrode $R(x)$. More specifically, the sensor output can be viewed as the two-sided Laplace transformed $R(x)$ multiplied by the strain measured at the coordinate origin. To emphasize the importance of this coordinate origin with respect to the $P o D$ sensor designed, the coordinate origin utilized here is termed a 'targeted origin'. It should be noted that once the method of image is adopted, the targeted origin can be placed anywhere within the sensor structure [3]. A detailed discussion and manipulation of the targeted origin have been previously given by Hsu and Lee in 2002 [3]. It was shown that there are two categories of $A P R O P O S$ device, i.e., an even-function $A P R O P O S$ device and an odd-function $A P R O P O S$ device. The terms 'even function' and 'odd function' were used to signify the characteristics of the spatial weighting function $R(x)$ with respect to the targeted origin in the spatial domain. These characteristics can be understood from figures 4(a) and (b), where figure 4(a) shows the schematic of an even-function APROPOS device with its targeted origin set at the free end, and figure 4(b) shows an odd-function APROPOS device with its targeted origin set at the fixed end. As detailed in a previous report [3] an even-function $A P R O P O S$ device can introduce a no-phase delay low-pass filter into a sensor transfer function, and an odd-function APROPOS device can also introduce a no-phase delay low-pass filter into a sensor transfer function with additional zeros. A more detailed discussion regarding the physical meaning of these sensors is detailed herein.

The sensor equations of the APROPOS device expended by the method of image in an infinite domain are [3]

$$
\begin{aligned}
q_{\mathrm{e}}(t) & =-z_{k} e_{31} \mathrm{e}^{\mathrm{j} \omega t} k^{2} \int_{-\infty}^{\infty} R_{\mathrm{e}}(x)\left[-\left(w_{\mathrm{lp}} \mathrm{e}^{\mathrm{j} k x}+w_{\mathrm{rp}} \mathrm{e}^{-\mathrm{j} k x}\right)\right. \\
& \left.+\left(w_{\mathrm{le}} \mathrm{e}^{k x}+w_{\mathrm{re}} \mathrm{e}^{-k x}\right)\right] \mathrm{d} x
\end{aligned}
$$

and 


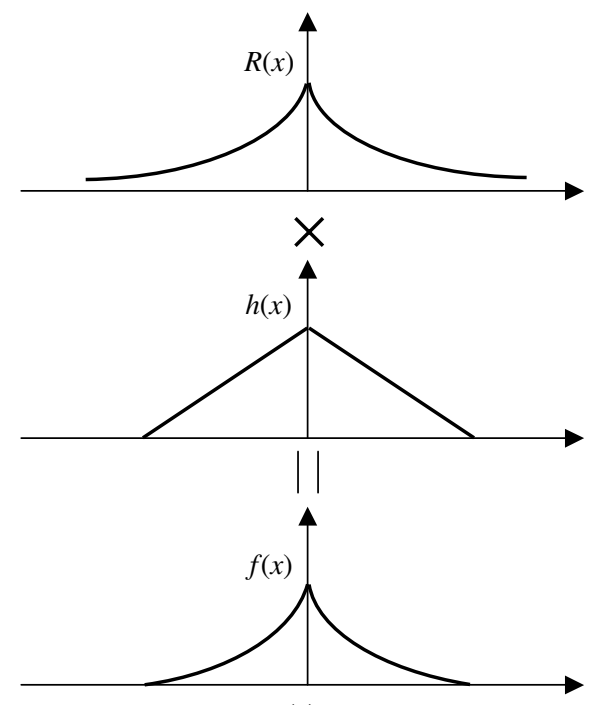

(a)

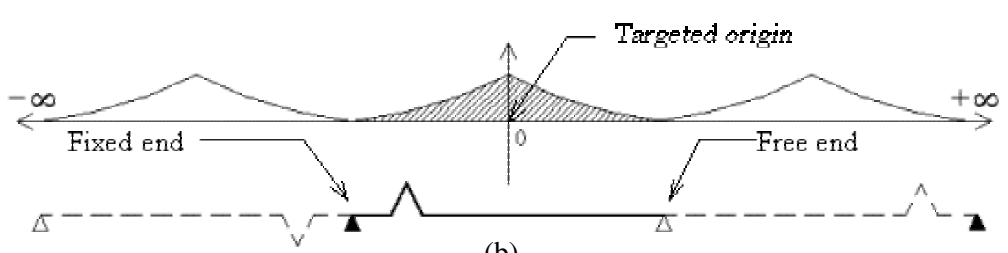

(b)

Figure 5. (a) Schematic of an APROPOS device designed using a window function. (b) Wave mode expansions on a cantilever plate by implementing method of image and an APROPOS device designed by using a window function.

$$
\begin{aligned}
q_{\mathrm{o}}(t) & =-z_{k} e_{31} \mathrm{e}^{\mathrm{j} \omega t} k^{2} \int_{-\infty}^{\infty} R_{\mathrm{o}}(x)\left[-\left(w_{\mathrm{lp}} \mathrm{e}^{\mathrm{j} k x}+w_{\mathrm{rp}} \mathrm{e}^{-\mathrm{j} k x}\right)\right. \\
& \left.+\left(w_{\mathrm{le}} \mathrm{e}^{k x}+w_{\mathrm{re}} \mathrm{e}^{-k x}\right)\right] \mathrm{d} x,
\end{aligned}
$$

where $q_{\mathrm{e}}(t)$ and $q_{\mathrm{o}}(t)$ represent the sensor equation of an even-function APROPOS device and odd-function APROPOS device, and $R_{\mathrm{e}}(t)$ and $R_{\mathrm{o}}(t)$ represent their weighting functions. It has been reported by Hsu and Lee that the sensor equation of the even-function $A P R O P O S$ device is [3]

$$
q_{\mathrm{e}}(t)=-z_{k} e_{31} \mathrm{e}^{\mathrm{j} \omega t} k^{2}\left[-\left(w_{\mathrm{lp}}+w_{\mathrm{rp}}\right)+\left(w_{\mathrm{le}}+w_{\mathrm{re}}\right)\right] G_{\mathrm{e}}(\omega),
$$

where $G_{\mathrm{e}}(\omega)$ is a no-phase delay low-pass filter in the spatial domain. Note that the physical quantity measured by the even-function $A P R O P O S$ device is a mechanical strain of the targeted origin. This can be easily derived by substituting zero into the $x$ of the second derivative of equation (4). The physical quantity measured by an odd-function APROPOS device appears to be ambiguous as additional zeros were introduced by using an odd-function effective surface electrode. It can be shown that these zeros incorporate the physical meaning intrinsically. From mathematical calculus we know that the derivative of an odd function is an even function, and vice versa. As such, an odd function $R_{\mathrm{o}}(x)$ can be written as $R_{\mathrm{e}}^{\prime}(x)$. The integration by parts to equation $(6 b)$ leads to

$$
\begin{aligned}
& q_{k}(t)=-z_{k} e_{31} \int_{-\infty}^{\infty} R_{\mathrm{e}}^{\prime}(x) \frac{\partial^{2} w}{\partial x^{2}} \mathrm{~d} x \\
& =-z_{k} e_{31}\left[\left.\left(R_{\mathrm{e}}^{\prime}(x) \frac{\partial^{2} w}{\partial x^{2}}\right)\right|_{-\infty} ^{\infty}-\int_{-\infty}^{\infty} R_{\mathrm{e}}(x) \frac{\partial^{3} w}{\partial x^{3}} \mathrm{~d} x\right] .
\end{aligned}
$$

Note that structure information is expressed as $w$, which is the bending displacement. As the spatial weighting introduced by the surface electrode is zero in an infinite domain, the boundary term in equation $(8 a)$ is zero. Thus the sensor equation of the odd-function $A P R O P O S$ device is

$$
\begin{aligned}
& q_{k}(t)=z_{k} e_{31} \int_{-\infty}^{\infty} R_{\mathrm{e}}(x) \frac{\partial^{3} w}{\partial x^{3}} \mathrm{~d} x \\
& =z_{k} e_{31} \mathrm{e}^{\mathrm{j} \omega t} k^{2} \int_{-\infty}^{\infty} R_{\mathrm{e}}(x)\left[-\mathrm{j} k w_{\mathrm{lp}} \mathrm{e}^{\mathrm{j} k x}+\mathrm{j} k w_{\mathrm{rp}} \mathrm{e}^{-\mathrm{j} k x}\right. \\
& \left.\quad+k w_{\mathrm{le}} \mathrm{e}^{k x}-k w_{\mathrm{re}} \mathrm{e}^{-k x}\right] \mathrm{d} x
\end{aligned}
$$

That is, the odd-function APROPOS device offers an evenfunction weighting to the shear force $\partial^{3} w / \partial x^{3}$ of the targeted origin in an infinite domain. The zeros previously mentioned by Hsu and Lee [3] are actually contributed by the local characteristics of the shear force at the targeted origin. Mathematically, the odd function introduced by the APROPOS device in a spatial domain offers a spatial derivative to the mechanical strain and ensures that the measured physical quantity is the mechanical shear force. As the weighting function is again an even function, the sensor equation of the odd-function APROPOS device can be simplified to

$$
q_{k}(t)=z_{k} e_{31} \mathrm{e}^{\mathrm{j} \omega t} k^{2}\left[-\mathrm{j} k w_{\mathrm{lp}}+\mathrm{j} k w_{\mathrm{rp}}+k w_{\mathrm{le}}-k w_{\mathrm{re}}\right] G_{\mathrm{o}}(\omega),
$$

where $G_{\mathrm{o}}(\omega)$ is the no-phase delay low-pass filter introduced by the odd-function $A P R O P O S$ device. Thus, the transfer function of a fixed-end APROPOS device is the superposition of the overall structure characteristic, the local information of the shear force at the fixed end, and the no-phase delay lowpass filter introduced by the APROPOS device. 
In addition to using the concept of method of image to map the wave modes into an infinite domain [3], the concept of a window function serves as another mathematical tool to deal with discontinuous waves at any arbitrary boundary (figure 5). To overcome this limitation, a window function [15] was introduced. To handle the case where the wave modes are discontinuous at the boundary points, window function $h(x)$ was multiplied to the effective surface electrode $R(x)$ such that the product becomes zero at the boundary (figure 5(a)). With this approach, the boundary effects are thus completely eliminated. The sensor equation of APROPOS devices designed by utilizing a windows function can be expressed as

$$
\begin{aligned}
q(t) & =-z_{k} e_{31} \mathrm{e}^{\mathrm{j} \omega t} k^{2} \int_{0}^{l} f(x)\left[-\left(w_{\mathrm{lp}} \mathrm{e}^{\mathrm{i} k x}+w_{\mathrm{rp}} \mathrm{e}^{-\mathrm{i} k x}\right)\right. \\
& \left.+\left(w_{\mathrm{le}} \mathrm{e}^{k x}+w_{\mathrm{re}} \mathrm{e}^{-k x}\right)\right] \mathrm{d} x,
\end{aligned}
$$

where

$$
f(x)=h(x) R(x),
$$

$h(x)$ is the window function multiplied to tailor the surface electrode $R(x)$, and the real effective surface electrode to be implemented becomes $f(x)=h(x) R(x)$. Thus, the utility of the weighting function $f(x)$ in equation (10) is identical to $R(x)$ in equation (5). Therefore, the limitations caused by the boundaries of the finite structure can be dealt with by the introduction of a method of image and a windows function, and where the waves propagating between the boundaries can be expanded into the infinite domain.

With the two methods mentioned above, the wave modes experienced by APROPOS devices can be made continuous in the infinite domain and can thus be expressed by taking the following two-sided Laplace transforms [3]. The transform variables $s$ for the propagating and evanescent waves are $-\mathrm{j} k$ and $-k$, respectively. Thus, the sensor equation becomes

$$
\begin{aligned}
q(t) & =-z_{k}^{0} e_{31} \mathrm{e}^{\mathrm{j} \omega t} k^{2}\left[-\left(w_{\text {lp }}+w_{\text {rp }}\right) \int_{-\infty}^{\infty} \mathbb{R}(x) \mathrm{e}^{-\mathrm{j} k x} \mathrm{~d} x\right. \\
& \left.+\left(w_{\text {le }}+w_{\text {re }}\right) \int_{-\infty}^{\infty} \mathbb{R}(x) \mathrm{e}^{-k x} \mathrm{~d} x\right]
\end{aligned}
$$

if effective surface electrode $\mathbb{R}(x)$ represents the weighting function $R(x)$ and $f(x)$ shown in equations (5) and (9), and where the integrants are symmetric with respect to the origin. That is, if the fixed end is placed at the origin, $\mathbb{R}(x)$ must be anti-symmetric with respect to the origin in an infinite domain. A $180^{\circ}$ phase change exists for the reflected wave that is expanded to the infinite domain. On the other hand, if a free end is chosen as the origin, $\mathbb{R}(x)$ must be symmetric with respect to the origin. In addition, the spatial filtering effects created by $\mathbb{R}(x)$ are superimposed onto the wave mode amplitudes as shown in equation (11). More specifically, equation (11) demonstrates that $A P R O P O S$ devices will not alter the original structure characteristics of $w_{\mathrm{lp}}, w_{\mathrm{rp}}, w_{\mathrm{le}}$, and $w_{\text {re }}$. Furthermore, since the two integrals of equation (11) are two real numbers, i.e., not imaginary, a no-phase delay filtering action is introduced by the weighting function $\mathbb{R}(x)$. Recall that the four wave mode amplitudes represent the local characteristic at $x=0$ (equation (4)), which is the origin of the two-sided Laplace transform. As all the above discussions include the symmetric point targets towards this origin, it is of particular importance for the design of an APROPOS device and is thus named as the targeted origin [3].

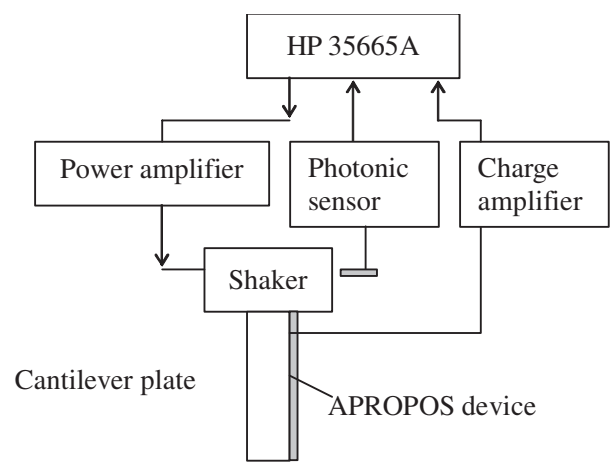

Figure 6. Schematic of the experimental set-up.

\subsection{Implementation of an APROPOS device by a windows function}

Consider the case of an APROPOS device (figure 1) which is placed on a one-dimensional cantilever plate and has its targeted origin set at the middle of the plate. Choose an exponential function $R(x)=\mathrm{e}^{-\alpha|x|}$ as the basic spatial filtering function, and where window function $h(x)=1-|x| / a$ nulls the weighting on both boundaries with $a$ as a half length of the one-dimensional cantilever plate. Substituting the spatial weighting function $\mathbb{R}(x)$, which is designed as a product of $R(x)$ and $h(x)$, into equation (11), the sensor equation becomes

$$
\begin{aligned}
q(t) & =-z_{k}^{0} e_{31} \mathrm{e}^{\mathrm{j} \omega t} k^{2} \frac{2}{a} \\
& \times\left[-\left(w_{\mathrm{lp}}+w_{\mathrm{rp}}\right) \frac{(a \alpha-1) \alpha^{2}+(a \alpha+1) k_{\mathrm{p}}^{2}}{\left(\alpha^{2}+k_{\mathrm{p}}^{2}\right)^{2}}\right. \\
& \left.+\left(w_{\mathrm{le}}+w_{\mathrm{re}}\right) \frac{(a \alpha-1) \alpha^{2}-(a \alpha+1) k_{\mathrm{e}}^{2}}{\left(\alpha^{2}-k_{\mathrm{e}}^{2}\right)^{2}}\right],
\end{aligned}
$$

where $k_{\mathrm{p}}$ and $k_{\mathrm{e}}$ are the wavenumbers of the propagating and evanescent waves, $\alpha$ is the corner wavenumber, and $a$ is the half length of the APROPOS device. Substituting the dispersion relationship shown in equation $(3 b)$ into (12), which are the dispersion relationships for propagating and evanescent waves, we obtain

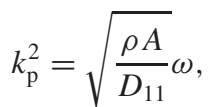

and

$$
-k_{\mathrm{e}}^{2}=\sqrt{\frac{\rho A}{D_{11}}} \omega,
$$

and where the sensor equation can be further simplified into

$$
q(t)=-z_{k}^{0} e_{31} \mathrm{e}^{\mathrm{j} \omega t} k^{2} \frac{2}{a}\left[-\left(w_{\mathrm{lp}}+w_{\mathrm{rp}}\right)+\left(w_{\mathrm{le}}+w_{\mathrm{re}}\right)\right] F(\omega),
$$

with

$$
F(\omega)=\frac{(a \alpha-1) \alpha^{2}+(a \alpha+1) \omega}{\left(\alpha^{2}+\omega\right)^{2}} .
$$

It is clear from equation ( $12 b)$ that the $A P R O P O S$ device can introduce a $-20 \mathrm{~dB} /$ decade no-phase delay low-pass filter into the frequency domain when $a \alpha>1$. To verify this concept, an experimental set-up (figure 6) with experimental conditions identical to the previous set-up by Hsu and Lee in 2002 [3] was performed. The one-dimensional plate was made of a $140 \mathrm{~mm}$ 


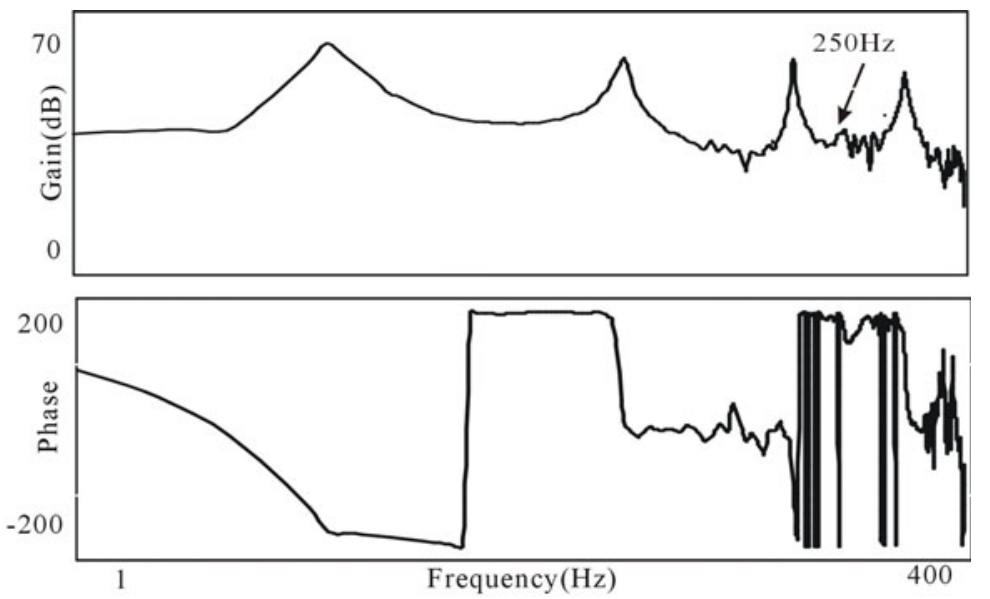

(a)

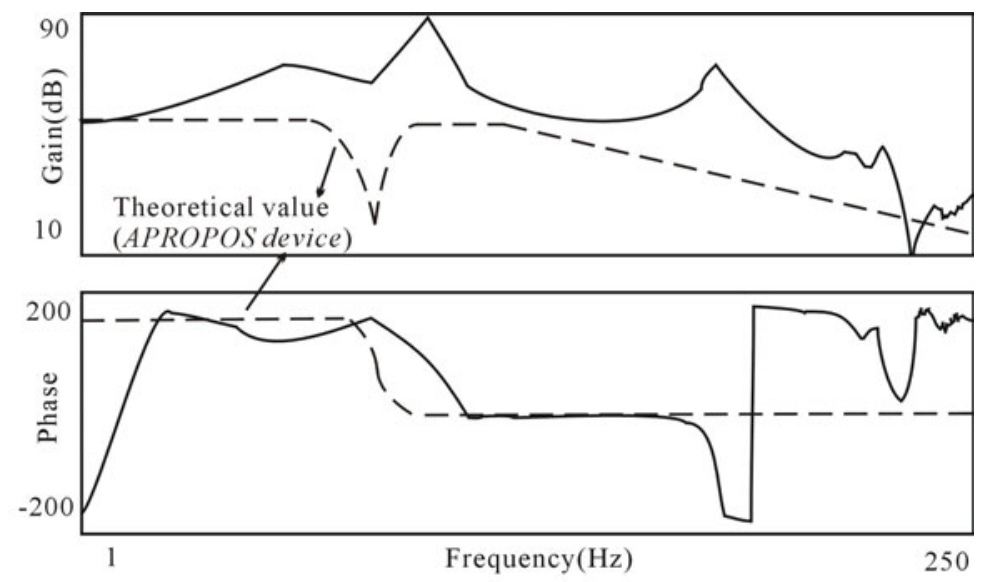

(b)

Figure 7. (a) Transfer function of a distributed sensor with uniform surface electrode. (b) Transfer function of a no-phase delay low-pass filter introduced by using the APROPOS device designed using a window function.

long by $22 \mathrm{~mm}$ wide by $0.02 \mathrm{~mm}$ thick stainless steel shim with the corner wavenumber of the APROPOS device chosen to be $k=10$ in a spatial domain. The corresponding corner frequency was thus equivalent to $32 \mathrm{~Hz}$, which was retrieved by using the dispersion relationship derived from the experimental transfer function of the uniform sensor shown in figure 7(a). The induced filter $F(\omega)$ can then be written as

$$
f(\omega)=-48.571 \frac{(5-\omega)}{(32+\omega)^{2}}
$$

where an extra zero around $5 \mathrm{~Hz}$ occurs. This zero is induced due to the fact that the product $a \alpha=0.7$ is less than one. This shows that the filtering effect of the APROPOS device designed by utilizing a window function must be carefully calculated to study its contribution to the sensor transfer function. The experimental result of this particular APROPOS device is shown in figure 7(b). It can be observed from figure 7(b) that a no-phase delay low-pass filter exists in a sensor transfer function when compared to the transfer function of a uniform sensor (figure 7(a)). The additional zeros introduced offer a $-180^{\circ}$ phase lag below $5 \mathrm{~Hz}$ and no additional phase contribution was found even at a frequency several hertz above its corner frequency. In addition, the no-phase delay low-pass filter induced by the low-pass filter $1 /(32+\omega)^{2}$ (equation $(12 c)$ ) introduces no additional phase information into the transfer function throughout the full frequency range. The dashed curve shown in figure 7(b) represents the theoretical value of the no-phase delay filter introduced by adopting the APROPOS device. These data agree well with the theoretical predictions (equation (12c)).

As the cantilever plate and the piezoelectric sensor are comparable in size, this agreement proves that the APROPOS device can circumvent the limitations faced by the spatial filter by taking into account the contributions of the evanescent waves and boundary effects. In other words, the APROPOS device concept can be adopted to facilitate a $P o D$ sensor implementation. Next, we will detail a micromachining-based free-fall sensor implemented by adopting a $P O D$ sensor design concept to further illustrate the potential of using an APROPOS device concept to vastly expand the design freedom of today's piezoelectric sensors, which certainly include that of MEMS devices.

\subsection{Miniature free-fall sensors}

The majority of impact detection studies have concentrated on detecting the information generated by an impact wave 


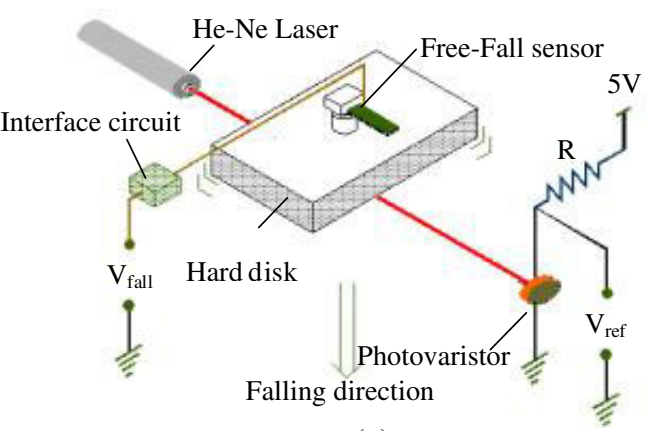

(a)

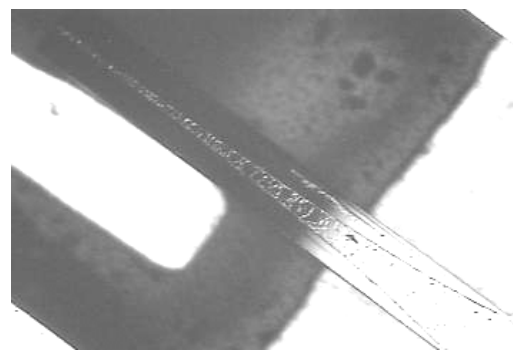

(b)

Figure 8. (a) Experimental set-up for measuring free-fall motion. (b) Micromachined-based free-fall sensor designed by an APROPOS device.

(This figure is in colour only in the electronic version)

induced when the specimen hits the ground. It is expected that a system controller can provide the evasive action needed for protection [19] by adopting the impact signals detected. As the design goal of today's portable devices are made smaller and faster, the impact protection issue is becoming increasingly more important. A simple analysis shows that sensing the start of the free fall and not the impact wave must be attempted to make this protection feature viable to today's data storage devices such as notebook computers, digital still cameras, etc. Taking hard disk drives as an example, the seek time that measures the time needed for the read-write head to move $3 \mathrm{~cm}$ is approximately $10 \mathrm{~ms}$. An impact-induced elastic wave has a propagation speed in the range of $3000-5000 \mathrm{~m} \mathrm{~s}^{-1}$. This speed indicates that the time between the impact and the read-write head experiencing the impact-induced wave is in the range of $10-30 \mu \mathrm{s}$, which is much shorter than the $10 \mathrm{~ms}$ mentioned above. Thus the signal obtained after the impact occurs will be too late to allow the system to take evasive action. The overall strategy related to the impact detection thus must be revisited. Considering the case where a data storage device falls from a height of $1 \mathrm{~m}$, the time difference between the start of the device falling to impact is around $450 \mathrm{~ms}$, which is much longer than the $10 \mathrm{~ms}$ seek time available. This simple calculation indicates that a free-fall sensor will have a much better chance of accomplishing the self-protection tasks mentioned above. The key idea behind a free-fall sensing as introduced herein lies in the fact that the acceleration changes from at rest to a gravity constant $g$ [20] at the beginning of the free fall. That is, the free-fall motion can be represented by a force function that starts from zero to one gravity. In other words, the design of the free-fall sensor needs to detect the rise of the acceleration associated with the sensor structure transient response driven by the gravitational force change.
To test the free-fall motion, an experimental set-up (figure 8(a)) was designed to measure the induced signal by Hsu and Lee [21]. Two free-fall sensors were implemented to detect the free-fall motion. The first was a $20.1 \mathrm{~mm}$ long, $6 \mathrm{~mm}$ wide, and $1.8 \mathrm{~mm}$ thick PZT with uniform electrodes, and the other one was a $2000 \mu \mathrm{m}$ long by $20 \mu \mathrm{m}$ wide by 6-7 $\mu \mathrm{m}$ thick micromachined-based free-fall sensor designed to become a $P o D$ sensor (figure 8(b)), where $\mathrm{ZnO}$ was used as its sensing material. The resonant frequencies of the PZT and $\mathrm{ZnO}$ sensors were 1.41 and $1.29 \mathrm{kHz}$, respectively. Following the design thinking mentioned above, an APROPOS device with its targeted origin set at the fixed end was applied to the $\mathrm{ZnO}$ sensor. The reason for putting the targeted origin of the APROPOS device designed for a free-fall sensor at the fixed end can be attributed to the fact that the vibration was induced through the fixed end. This design provides the APROPOS device with a capability to measure the free-fall motion at the time it starts without considering the wave propagation delay associated with the finite length of the sensor structure. The corner wavenumber of the PoD sensor was set at 0.1 by choosing effective surface electrode $R(x)=\mathrm{e}^{-0.1|x|}-$ $0.5712 \sin (0.01122 x)$, which corresponds to a $14.698 \mathrm{~Hz}$ corner frequency by utilizing the dispersion relationship shown in equation $(3 b)$. The exponential function $\mathrm{e}^{-0.1|x|}$ offers a spatial filter effect to the sensor transfer function since the function is used to null the non-zero exponential function at the free end with a weighting factor. The utilization of the sine function is due to the fact that a two-sided Laplace transform of a sine function in an infinite domain is also zero. Recall that the schematics shown in figures 4(a) and (b) where the APROPOS device with the waves in an infinite domain is an even function when the targeted origin is placed on the free end and it is an odd function if the targeted origin is placed on the fixed end [3]. Substituting the weighting function of the effective surface electrode into equation $(8 b)$, the transfer function of this fixed-end APROPOS device is

$$
q_{k}(t)=z_{k} e_{31} w^{\prime \prime \prime}(x=0, t) \frac{1}{14.698+\omega},
$$

where a no-phase delay low-pass filter with corner frequency at $14.698 \mathrm{~Hz}$ is introduced to the transfer function of the measured shear force at the fixed end.

Both free-fall sensors were attached to a piece of a hard disk shaped iron block for testing. The current amplifier with a built-in electronic one-pole $10 \mathrm{kHz}$ low-pass filter was chosen to interface the free-fall sensors. The photovaristor resistor $R$, which changes with the amount of incident light, was placed into a voltage divider circuit to provide the trigger signal for the oscilloscope. During the experimental set-up, the test specimen fell through a light switch made of a He-Ne laser and changed the photovaristor $R$ value. The normalized transfer functions obtained by setting the resonant frequencies of these two sensors identical are shown in figure 9, where the thick and the thin curves represent the PZT plate and the micromachinedbased $P O D$ sensor, respectively. Note that the low-pass filter introduced from the spatial domain by using the APROPOS device reduces the influence of the first resonance mode of the micromachined-based free-fall sensor (thin curve in figure 9) without sacrificing the phase information. This is different from that of the PZT plate output as used in external electrical filters to eliminate first resonance mode contributions. 

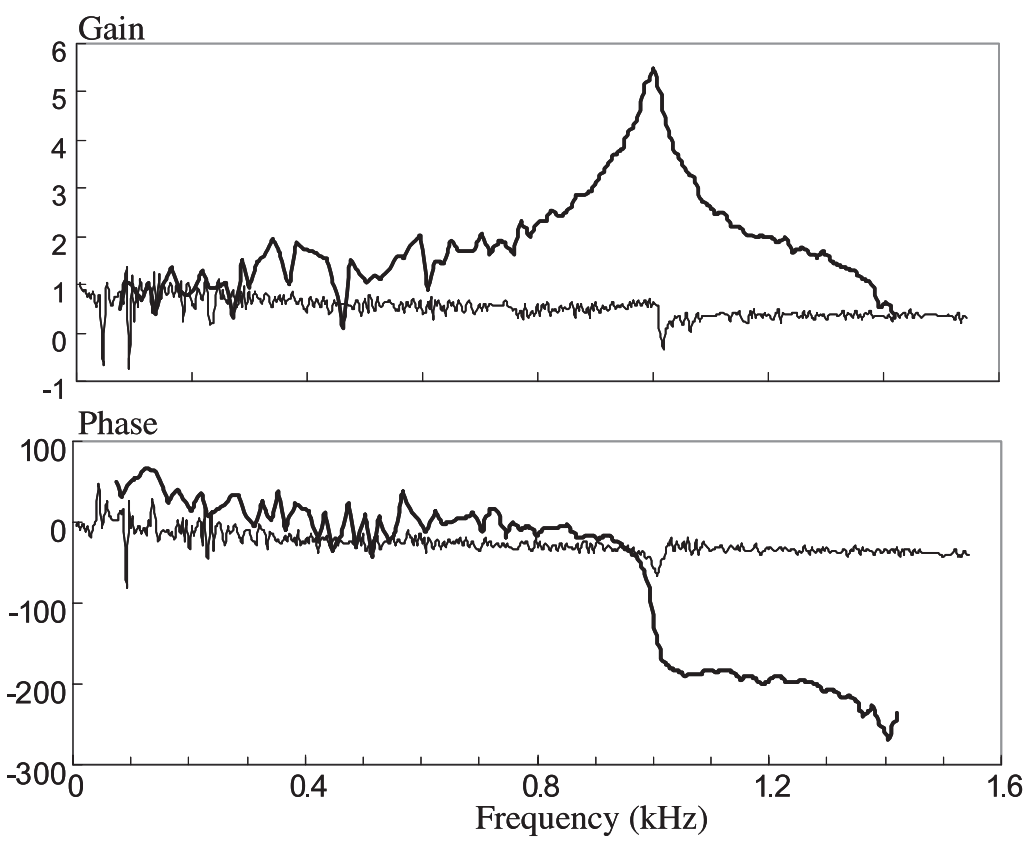

Figure 9. Normalized transfer function of (1) PZT plate (thick curve) and (2) micromachined-based PoD sensor (thin curve).

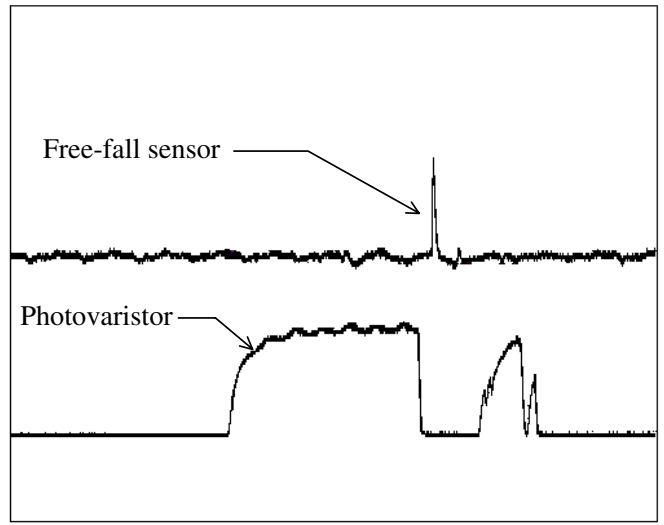

(a)

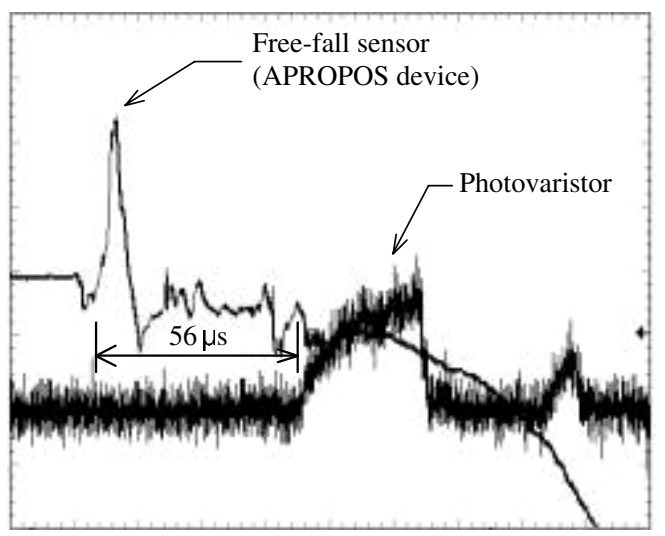

(b)

Figure 10. (a) Experimental result of a current-mode free-fall sensor with uniform electrode and signal conditioned with an externally supplied $-40 \mathrm{~dB} /$ decade electrical low-pass filter. (b) Experimental results of a current-mode free-fall PoD sensor.

Figure 10 shows the experimental results of these two freefall sensors obtained by using current amplifiers as the interface circuits. The distance between the He-Ne laser to the starting point of free-fall $h$ was found to be around $1.7 \mathrm{~cm}$, which corresponds to around $58.87 \mu \mathrm{s}$ as per the free-fall equation $h=g t^{2} / 2$ with $g=9.8 \mathrm{~m} \mathrm{~s}^{-2}$. Note that the micromachinedbased free-fall sensor measured free-fall motion at around $56 \mu$ s before the photovaristor was triggered. This result matches well with the theoretical value. On the other hand, the data obtained from the PZT plate that detected the free-fall motion a few microseconds later clearly confirms the inevitable phase lag associated with an electrical filter where the phase lag delays the measurement signal. This experiment demonstrates that a free-fall sensor can be implemented by using a $P o D$ sensor concept.

The spatial characteristics of the piezoelectric lamina shown above reveal a new-found freedom in designing piezoelectric sensors, which clearly demonstrates that the spatial characteristics of the sensor structure itself is an important design parameter to consider when pursuing innovative signal processing on signals to be obtained from piezoelectric systems. To further understand the advantages of a spatial relationship between sensor and actuator pairs including both shapes and locations, more studies were conducted.

\section{Pole-zero alternating phenomenon between sensor and actuator on a piezoelectric plate}

As was mentioned previously, Martin in 1978 reported on the pole-zero alternating phenomenon of flexible structures [12], which states that the transfer function of a structure system under external excitation in terms of normal-mode $[12,22]$ is

$$
\frac{y(r, s)}{u(s)}=\sum_{i=1}^{N} \frac{h_{i} g_{i}}{\left(s^{2}+2 \zeta_{i} \omega_{i} s+\omega_{i}^{2}\right)} .
$$

In equation (14), $u$ is the input magnitude for a modal coordinate, $y$ is the measured mechanical motion at location 
$r, g_{i}$ is the row vector of the $i$ th mode participation factor of input, $h_{i}$ is a function of mode shape at $r, \omega_{i}$ and $\zeta_{i}$ are the resonance frequency of the $i$ th mode and damping ratio, and $s$ is equal to $\mathrm{j} \omega$. Equation (14) states that the transfer function of an excited structure system is actually the superposition of all structure modes. The excitation term $g_{i}$ represents how much the external force contributes to the $i$ th mode, and the sensing term $h_{i}$ represents how much the induced motion has been measured at the $i$ th mode. Martin proposed a corollary stating that there exists a zero at frequency $z_{i}$ between any two structural modes if and only if

$$
\operatorname{sgn}\left(h_{i} g_{i}\right)=\operatorname{sgn}\left(h_{j} g_{j}\right) \quad \text { for all } i, j .
$$

Since the sign of the $h_{i} g_{i}$ depends on the location of the excitation and the sensing points of the structure, the transfer function will depend on the placement of the actuator and sensor. To ensure the stability of a controlled flexible structure system, Martin proposed to adopt a co-located sensor and actuator pair configuration to ensure that the sign of $h_{i} g_{i}$ for each mode will be identical. In this way, the transfer function becomes pole-zero alternating throughout the frequency domain. This design algorithm enhances the phase margin of the structure system and makes flexible structure control easier to implement. In other words, the transfer function of a structure system is determined by the summation of the contributions of the sensor and actuator for each mode, as shown in equation (14). It should be noted that the pole-zero alternating phenomenon is a spatial effect and can be utilized to modify the transfer function by introducing properly designed distributed actuators and sensors, as well as point actuators and sensors.

The capability of being able to manipulate the pole-zero alternating phenomenon becomes a major design parameter for some current actively pursued piezoelectric technologies such as piezoelectric transformers [23] and piezoelectric gyroscopes [24], both of which operate in a predetermined resonance mode. The presence of multiple modes leads to densely packed resonance peaks in the transfer function. Contributions from unwanted resonance modes often cause major concerns in designing resonators as they invariably cause instability, introduce noise, or decrease device lifetime. Taking a one-dimensional plate with a free-free boundary condition as an example, the resonant mode appears every octave and is far denser than that of the roll-off effect of typical electrical filters. More specifically, since the roll-off behavior of a one-pole electronic filter is $20 \mathrm{~dB} /$ decade, it cannot filter out unwanted structure modes which appear at every octave, not to mention the phase delay associated with the introduction of the electronic filter. To operate at the pre-specified resonant frequency, resonant devices mentioned above have adopted various very complex interface circuits. No fundamental breakthrough has been found for this problem so far despite intensive research efforts invested over the years. It can be shown that by properly manipulating the polezero alternating phenomenon, the phase distributions and the occurrence of zeros can be utilized to tailor the transfer function between actuators and sensors. With this approach, innovative resonance piezoelectric system circuitry can be realized by adopting tailored actuator and sensor pairs.

\subsection{Pole-zero alternating phenomenon on piezoelectric} plates

Three configurations of piezoelectric plates were used to study the pole-zero alternating phenomenon of the transfer function between the sensor and actuator. Figure 11 shows the schematics of the piezoelectric plates, which are the configurations of (a) surface-to-surface, (b) side-by-side, and (c) adjoining piezoelectric plates. Only the longitudinal motions of these piezoelectric plates were used to study the pole-zero alternating phenomena between the actuator and sensor. The structure coordinate axes of these piezoelectric plates are also shown in figure 11 , which were chosen to match the material coordinate axes of the actuators. To explain this effect, a one-dimensional field equation of the piezoelectric plate was derived from the original partial differential equations (PDEs) [25]. The result is different from the equivalent circuit model developed based on an ordinary differential equation (ODE) perspective [26]. Several assumptions made to simplify the three-dimensional governing equation to one-dimensional cases are discussed herein. These assumptions include assuming that

(1) the thickness of the piezoelectric plate $t$ is much thinner than its width $w$ and length $l$,

(2) the thickness of the electrodes is much thinner than the thickness of the piezoelectric plate $t$,

(3) the displacement fields are symmetric with respect to its neutral plane,

(4) length $l$ is much longer than width $w$, and

(5) the piezoelectric plates operate under a long-wavelength longitudinal motion only.

The fully coupled one-dimensional field equation of piezoelectric plates will be detailed based on these assumptions.

\subsection{One-dimensional governing equation of piezoelectric plates}

The basic configuration of the piezoelectric plates shown in figure 11 can be categorized into two kinds of piezoelectric plates. The two types of piezoelectric plates are shown in figures 12(a) and (b), which show their poling direction are along the thickness and the lateral direction. A $44 \mathrm{~mm}$ long by $6.5 \mathrm{~mm}$ wide by $2.2 \mathrm{~mm}$ thick PZT (lead, zirconate, and titanate) plate was used as the test structure; the PZT possessed class $6 \mathrm{~mm}$ symmetry. The governing equation and the constitutive equation of the one-dimensional plates as shown in figures 12(a) and (b) can be reduced from the original threedimensional partial differential equations to $(12 a)$ and $(12 b)$ respectively [27]:

$$
\begin{gathered}
c_{11}^{E} \tilde{u}_{1,11}-e_{31} V_{3}^{\text {in }}=\rho \tilde{\ddot{u}}_{1} \\
\tilde{T}_{1}=c_{11}^{E} \tilde{u}_{1,1}-e_{31}\left(V_{3}\right) \\
t \tilde{D}_{3}=e_{31} \tilde{u}_{1,1}+\varepsilon_{33}^{s}\left(V_{3}\right),
\end{gathered}
$$

and

$$
\begin{gathered}
\left(c_{33}^{E}+e_{33}^{2} / \varepsilon_{33}^{s}\right) \tilde{u}_{3,33}=\rho \tilde{\ddot{u}}_{3} \\
\tilde{T}_{3}=c_{33}^{E} \tilde{u}_{3,3}-e_{33}\left(\tilde{E}_{3}\right) \\
\tilde{D}_{3}=e_{33} \tilde{u}_{3,3}+\varepsilon_{33}^{s}\left(\tilde{E}_{3}\right),
\end{gathered}
$$




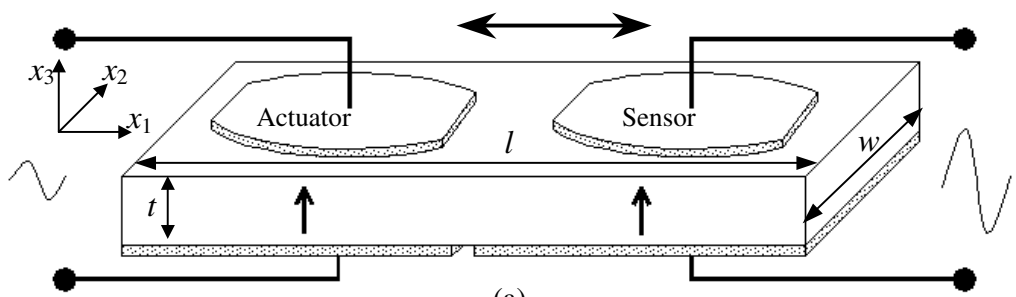

(a)

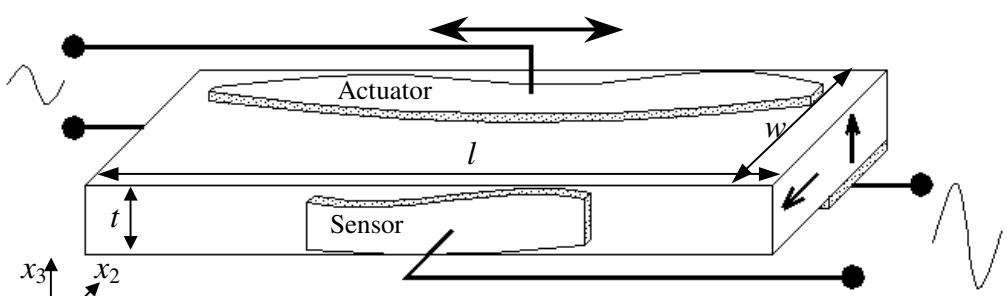

(b)

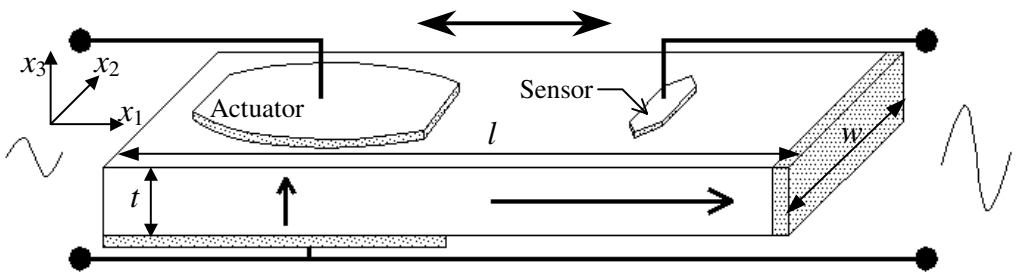

(c)

Figure 11. Schematic and structure coordinate axes of piezoelectric plates operating in a longitudinal motion: (a) surface-to-surface case, (b) side-by-side case, and (c) adjoining piezoelectric plates case.

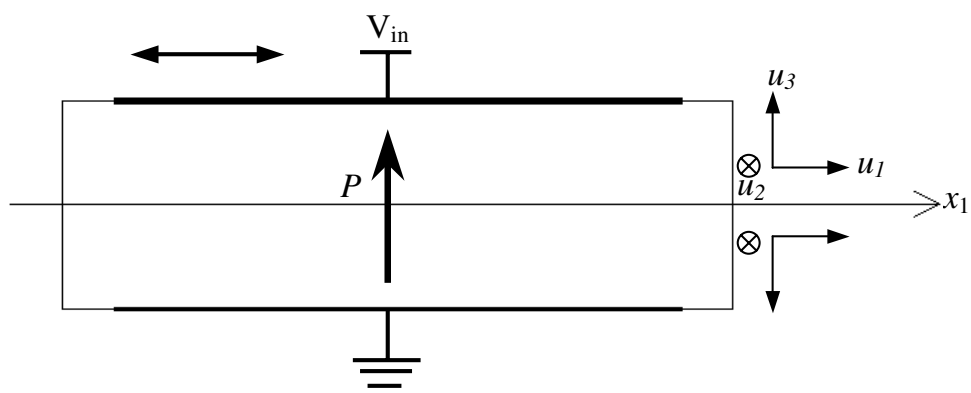

(a)

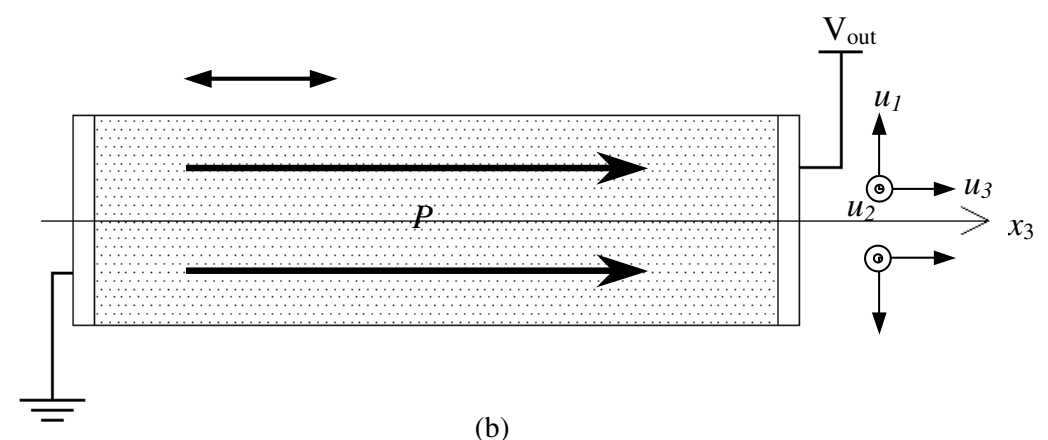

(b)

Figure 12. Schematic and axes of piezoelectric plates with poling direction in (a) a thickness direction and (b) a lateral direction.

where $u_{i, j}$ and $u_{i, j j}$ are the first and the second direction derivatives with respect to the $j$ th direction for displacement along the $i$ th direction, $T$ represents stress, $D$ and $E$ represent the electric displacement and electrical field, $c_{11}$ and $c_{33}$ are the elastic compliance constants, $\varepsilon$ is the permittivity constant, $e_{31}$ and $e_{33}$ are the piezoelectric stress constants, the superscripts ' $S$ ' and ' $E$ ' indicate that the $S$ and $E$ fields remain constants, $V$ is the applied voltage, $t$ is the thickness, and the sign ' $\sim$ ' indicates that the physical quantity is integrated along the thickness direction that is 
different for the two types of piezoelectric plates shown in figure 12 [27].

Note that these two one-dimensional governing equations are different due to their different poling direction. Piezoelectric constants $e_{31}$ and $e_{33}$ are used to properly represent the differences of the poling direction when considering the longitudinal vibration of the piezoelectric plate. It is clear from equation $(16 a)$ that piezoelectric effects serve as an inhomogeneous term for thickness poling piezoelectric plates. In addition, equation $(16 b)$ indicates that piezoelectric effects stiffen the elastic constant of the structure when the poling is along the lateral direction, i.e., the mechanical axis $z$. It should be mentioned that the stiffened elastic constant shown in equation $(16 b)$ is equal to $c_{33}^{D}$ when we consider the relationship between the material constants [25]. Examining the longitudinal structure motion, we find that the governing equations for both the actuator and sensor of the surface-to-surface and side-by-side piezoelectric plates are equation (16a) since only the longitudinal motion was considered. The $x_{3}$ axis was conventionally chosen as the poling direction of the piezoelectric material. This governing equation is also applicable to the actuator side of the adjoining piezoelectric plate if equation $(16 b)$ is adopted for the sensor side of the adjoining piezoelectric plate.

The general solution derived from normal mode expansion for both surface-to-surface and side-by-side piezoelectric plates using equation $(16 a)$ becomes

$$
\tilde{u}(x, t)=\sum_{i=1}^{\infty} A_{i}(t) \phi_{i}(x)=\sum_{i=1}^{\infty} \frac{r_{i}}{\left(\omega_{i}^{2}-\omega^{2}\right)} \phi_{i}(x),
$$

where

$$
\begin{aligned}
r_{i}(t) & =-\frac{e_{31}}{\rho} \int_{0}^{l}\left[V_{3}^{\text {in }} A_{\mathrm{a}}^{\prime}(x)+V_{3}^{\text {out }} S_{\mathrm{s}}^{\prime}(x)\right] \phi_{i}(x) \mathrm{d} x \\
& +\frac{c_{11}^{E}}{\rho}\left[\frac{\partial \tilde{u}(l, t)}{\partial x} \phi_{i}(l)-\frac{\partial \tilde{u}(0, t)}{\partial x} \phi_{i}(0)\right],
\end{aligned}
$$

$A_{\mathrm{a}}(x)$ and $S_{\mathrm{s}}(x)$ are the shapes of the actuator and sensor electrodes, $\omega_{i}=\left(C_{11}^{E} k_{i}^{2} / \rho\right)^{1 / 2}$ is the $i$ th resonance frequency of the piezoelectric plate, and $\phi_{i}(x)$ is the $i$ th eigen function. As the mechanical boundary condition of the piezoelectric plates is traction-free on both free ends, the second term in equation (18) is zero. It is clear that the piezoelectric effect appears as an external force that is applied at the piezoelectric plate.

Different from the surface-to-surface and side-by-side piezoelectric plates, adjoining piezoelectric plates must be solved by merging the two governing equations (16a) and $(16 b)$. Since it is a single structure as a whole, its resonance frequency will be identical even though the wavelength will be different for each side. The following displacement field represents the general solution of the adjoining piezoelectric plate with $A_{\mathrm{a}}(x)$ where the shape of the effective surface electrode on the actuator sides is derived using a normal mode expansion:

$$
\tilde{u}(x, t)=\sum_{i=1}^{\infty} A_{i}(t) \phi_{i}(x)=\sum_{i=1}^{\infty} \frac{r_{i}}{\left(\omega_{i}^{2}-\omega^{2}\right)} \phi_{i}(x),
$$

where

$$
r_{i}=-\frac{e_{31}}{\rho} V_{3}^{\text {in }} \int_{0}^{l_{1}} A_{\mathrm{a}}^{\prime}(x) \phi_{i}(x) \mathrm{d} x+\frac{e_{33}}{\rho \varepsilon_{33}^{s}} \tilde{D}_{3}\left(l_{\mathrm{p}}, t\right) \phi_{i}^{s}\left(l_{\mathrm{p}}\right),
$$

$\omega_{i}=\left(C_{11}^{E} k_{i}^{2} / \rho\right)^{1 / 2}$ is the $i$ th resonance frequency of the piezoelectric plate, $l_{1}$ and $l_{\mathrm{p}}$ are the length of the actuator and the adjoining piezoelectric plate, and $\phi_{i}(x)$ is the eigenfunction of the merged governing equation. The transfer function of the piezoelectric plates will be detailed herein with the theoretical displacement fields shown in equations (17) and (19).

\subsection{Transfer function of piezoelectric plates}

Taking a surface integral with respect to the constitutive equation $(16 a)$ of the sensor in a surface-to-surface and sideby-side piezoelectric plate leads to the following transfer function between the sensor and actuator:

$$
\begin{aligned}
\frac{V_{3 \mathrm{~s}}^{\text {out }}}{V_{3 \mathrm{a}}^{\text {in }}} & =\sum_{i=1}^{\infty} \frac{\mathrm{j} \omega Z_{\mathrm{L}} C_{\mathrm{s}}}{1+\mathrm{j} \omega Z_{\mathrm{L}} C_{\mathrm{s}}} \\
& \times \frac{\frac{w}{h} \frac{e_{31}^{2}}{\rho C_{\mathrm{s}}} A_{1} S_{2}}{\left(\omega_{i}^{2}+2 \zeta(\mathrm{j} \omega) \omega_{i}-\omega^{2}\right)-\frac{w}{h} \frac{\mathrm{j} \omega Z_{\mathrm{L}} C_{\mathrm{s}}}{1+\mathrm{j} \omega Z_{\mathrm{L}} C_{\mathrm{s}}} \frac{e_{31}^{2}}{\rho C_{\mathrm{s}}} S_{1} S_{2}},
\end{aligned}
$$

where

$$
\begin{aligned}
\omega_{i}^{\prime 2}= & \omega_{i}^{2}-m_{\mathrm{a}} \frac{w}{t_{\mathrm{a}}} \frac{e_{31} e_{31}}{\rho C_{\mathrm{a}}} A_{1} A_{2}, \\
A_{1} & =\int_{0}^{l} A_{\mathrm{a}}^{\prime}(x) \phi_{i}(x) \mathrm{d} x, \\
S_{1} & =\int_{0}^{l} S_{\mathrm{a}}^{\prime}(x) \phi_{i}(x) \mathrm{d} x, \\
S_{2} & =\int_{0}^{l} S_{\mathrm{a}}(x) \phi_{i}^{\prime}(x) \mathrm{d} x,
\end{aligned}
$$

and $C_{\mathrm{a}}$ and $C_{\mathrm{s}}$ are the static capacitance of the actuator and sensor. This equation shows the transfer function between the sensor and actuator on a one-dimensional piezoelectric plate as a function of the mechanical and electrical parameters of the sensor and actuator. More specifically, the transfer function depends on (1) the location, (2) the shape of the surface electrodes, (3) the static capacitances of the sensor and actuator, and (4) the load impedance $Z_{\mathrm{L}}$. To examine the contribution due to the mechanical parameters, the load impedance connected to the piezoelectric plate was assumed to be infinite in comparison to the output impedance of the sensor, i.e., the output static capacitance. Under this assumption, the pole-zero alternating phenomenon of the piezoelectric plate and the transfer function will only be a function of the mechanical parameters, i.e., the generic properties associated with the construction of the piezoelectric plates. More specifically, the transfer function of surface-to-surface and side-by-side piezoelectric plates can be simplified to

$$
\frac{V_{3 \mathrm{~s}}^{\text {out }}}{V_{3 \mathrm{a}}^{\text {in }}}=\sum_{i=1}^{\infty} \frac{w}{h} \frac{e_{31}^{2}}{\rho C_{\mathrm{s}}} \frac{A_{1} S_{2}}{\left(\omega_{i}^{2}+2 \zeta(\mathrm{j} \omega) \omega_{i}-\omega^{2}\right)} .
$$

Following the same reasoning, the load impedance can also be assumed to be infinite when considering the transfer function of the adjoining piezoelectric plate case as

$$
\frac{V_{3 \mathrm{~s}}^{\text {out }}}{V_{3 \mathrm{a}}^{\text {in }}}=\sum_{i=1}^{\infty} \frac{w}{h} \frac{e_{31}^{2}}{\rho C_{\mathrm{s}}} \frac{A_{1} S_{3}}{\left(\omega_{i}^{2}+2 \zeta(\mathrm{j} \omega) \omega_{i}-\omega^{2}\right)},
$$




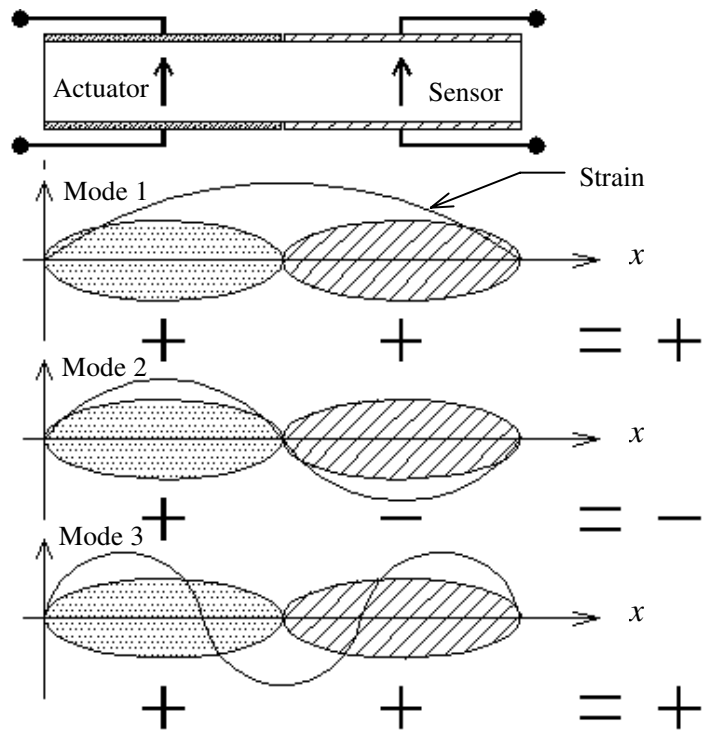

(a)

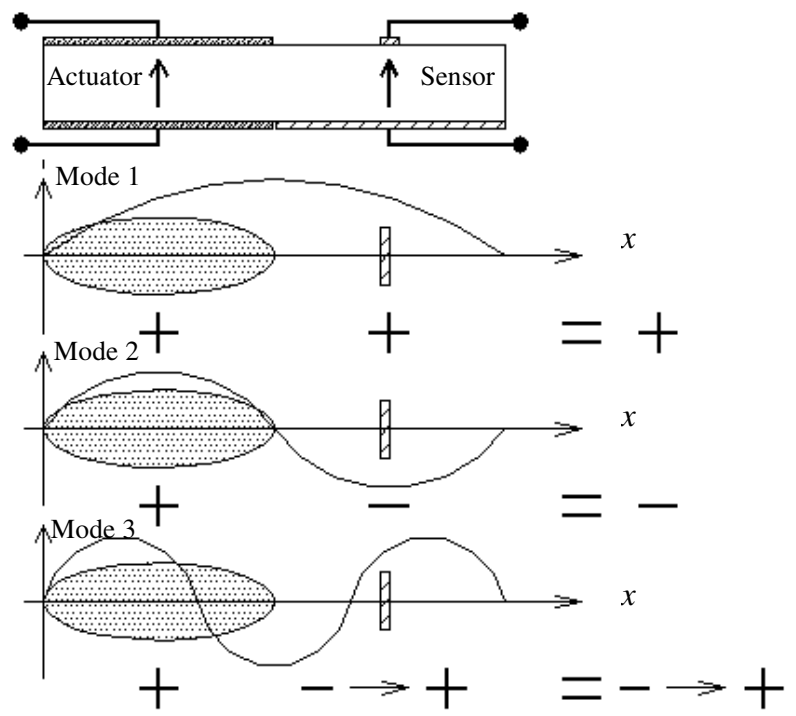

(b)

Figure 13. Schematic of the sign convention of the surface integral of a sensor and actuator on a surface-to-surface piezoelectric plate for (a) distributed actuator and sensor, and (b) distributed actuator and point sensor.

and

$$
S_{3}=\int_{0}^{l}\left(x-l_{1}\right) \phi_{i}^{\prime}(x) S_{\mathrm{s}}(x) \mathrm{d} x .
$$

Considering the derived transfer function of the piezoelectric plate as shown in equations (24) and (25) with the contributions of the actuator in the spatial integral as $A_{1}$, and noting the contribution of the sensors as $S_{1}$ and $S_{2}$, the spatial integral of $A_{1}, S_{1}$, and $S_{2}$ are clearly a result of performing surface integrals with respect to the $i$ th mode and with the electrodes as the integration domain per equations (23a), (23b) and (26). Following Martin's corollary there exists a zero between the two adjoining structure modes if and only if [12]

$$
\begin{aligned}
& \operatorname{sgn}\left(A_{1} S_{1}\right)^{i}=\operatorname{sgn}\left(A_{1} S_{1}\right)^{i+1}, \\
& \operatorname{sgn}\left(A_{1} S_{2}\right)^{i}=\operatorname{sgn}\left(A_{1} S_{2}\right)^{i+1},
\end{aligned}
$$

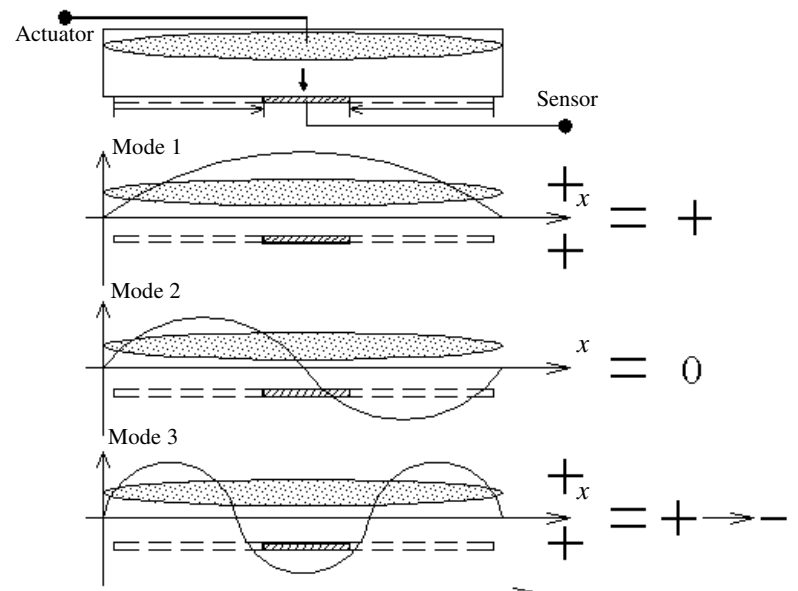

(a)

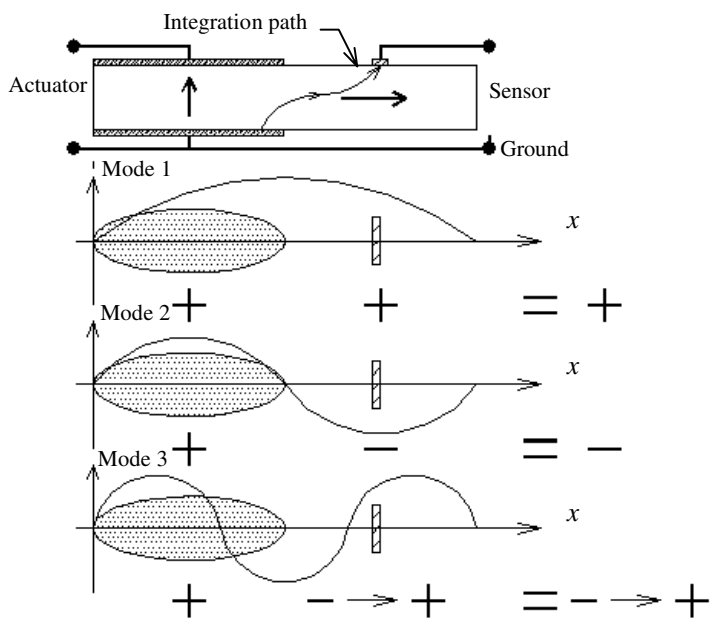

(b)

Figure 14. Schematic of the sign convention of a surface integral of a sensor and actuator for (a) a side-by-side piezoelectric plate with distributed actuator and sensor, and (b) an adjoining piezoelectric plate with a distributed actuator and point sensor.

where the superscripts $i$ and $i+1$ represent the two adjoining modes. Since all of the surface integrals of $A_{1}, S_{2}$, and $S_{3}$ are functions of the shape and the location placement of the sensors and actuators, it reveals that the spatial relationship between the sensor and actuator pairs serve as a tool in the transfer function. It should be noted that each of the actuators and sensors can also be distributed or pointed actuators and sensors.

Figures 13 and 14 show four experimental set-ups to study this physical phenomenon. Figures 13(a) and (b) use surface-to-surface piezoelectric plates to study the pole-zero alternating phenomenon for the case where the actuator and sensor are placed at different locations. Figure 13(a) shows the sign of the distributed actuator and sensor with each covering half of the piezoelectric plate. Note that the sign of the actuator will be positive for the first three resonance modes. In addition, the sign for the sensor will be positive for modes 1 and 3 and will be negative for the second mode. The resultant sign of the first three modes will thus be positive-negativepositive. Based on the understanding that the shapes of the sensor and actuator can alter the system transfer function as per equations (24) and (25), we know that even if arbitrary 

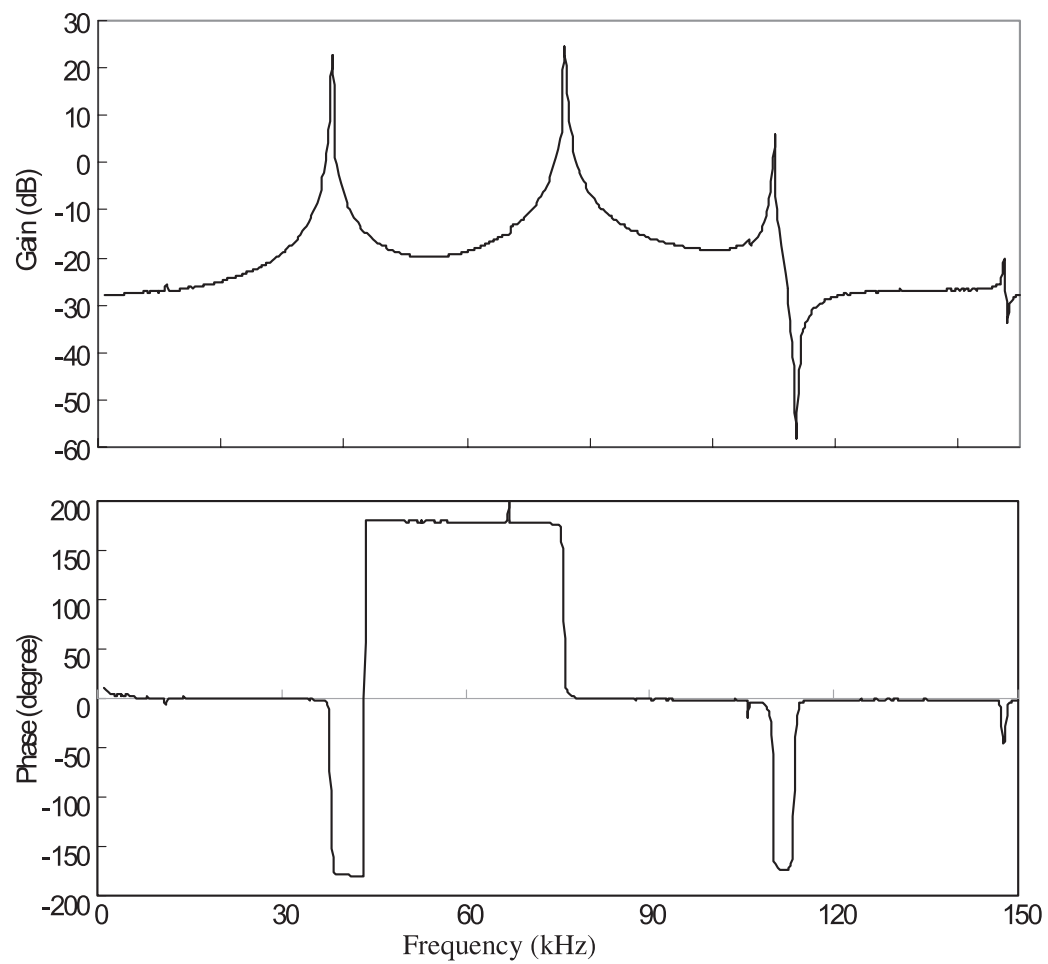

Figure 15. Transfer function of a quasi-mode-two actuator and sensor on a surface-to-surface piezoelectric plate.

shapes are chosen, the transfer function will be difficult to predict. Even in such cases where the transfer function is predictable, a fundamental understanding will be difficult to generate. The second reason is that a modal actuator and sensor offers zero weighting at the boundaries of the electrodes, which means that the electrical potential of the actuator and sensor on a surface-to-surface piezoelectric plate will not be coupled. Figure 15 shows the experimental result with its actuator and sensor designed as a quasi-modal actuator and sensor [8]. The result satisfies Martin's corollary which states that no zeros shall exist between the modes. In figure 13(b), we see the sign of a distributed actuator and a point sensor on a surface-tosurface piezoelectric plate with the location of the point sensor moved from the middle of the plate to the free end. From this figure, it is obvious that the sign of the sensor for mode 3 can vary from negative to positive, which means that a zero will exist between mode 2 and mode 3 if the sign of the sensor is negative. Figures 16(a) and (b) show the piezoelectric plate and the associated experimental result where the placement of the point sensor is tailored to the occurrence of the zero. Note that the point sensor is actually a symmetric distributed sensor to enhance the structure information of the targeted origin. The reason to adopt a symmetrically distributed sensor to mimic a point sensor lies in equations (23) as the electrode of (1) a rectangular point sensor $H(x-a)-H(x-s)$ and (2) a symmetric distributed sensor $\mathrm{e}^{-\alpha\left|x-x_{\mathrm{s}}\right|}[H(x-a)-H(x-b)]$ induce a boundary force and distributed force:

$$
\phi_{i}(b)-\phi_{i}(a)
$$

and

$$
\begin{aligned}
& \frac{1}{k_{i}^{2}-\alpha^{2}}\left\{2 \alpha \sin \left(k_{i} x_{\mathrm{s}}\right)-\mathrm{e}^{-b x}\left[\cos \left(k_{i} b\right)-\cos \left(k_{i} a\right)\right]\right. \\
& \left.\quad-\alpha \mathrm{e}^{-b x}\left[\sin \left(k_{i} b\right)-\sin \left(k_{i} a\right)\right]\right\} .
\end{aligned}
$$

It can be found that the measured two boundary forces by a rectangular point sensor as shown in equation (28a) will cancel each other out for the case where the electrode is too small, i.e., $b$ is close to $a$. This is not applicable to both the actuator and sensor, since driving forces or sensing intensity will disappear. On the other hand, the symmetric distributed sensor picks out the strain information of the location at point $x_{\mathrm{s}}$, and its boundary terms cancel each other out at the time $b$ is close to $a$. It is obvious from equations (28) that the symmetric distributed sensor really is a point sensor if the sensor size is small, and only the strain information from point $x_{\mathrm{s}}$ will be measured.

To study the case where the actuator and sensor are colocated, a side-by-side piezoelectric plate was used as the vehicle. Figure 14(a) shows the schematic of the distributed actuator and sensor on a side-by-side piezoelectric plate. The distributed sensor was a symmetric electrode with respect to the middle point of the plate and varied from the boundary to the center region (dashed line to solid line). Since the actuator was a symmetric electrode with respect to the center of the piezoelectric plate, the magnitude of the surface integral with respect to mode 2 will be zero for an anti-symmetric strain distribution such as that of mode 2. The pole-zero alternating phenomenon will thus depend on the sign of mode 1 and mode 3. Note that the sign contributed by the sensor will vary from positive to negative when the sensor electrode shrinks so as to let the negative strain dominate. Again, based on Martin's corollary, a zero will occur for a longer sensor electrode and will disappear for a smaller one. Figure 17 shows the experimental results where the zero next to mode 3 is switched from a lower frequency to a higher one.

Similarly, this effect also holds for an adjoining structure as shown in figure 14(b) which is different from figure 13(b) in 


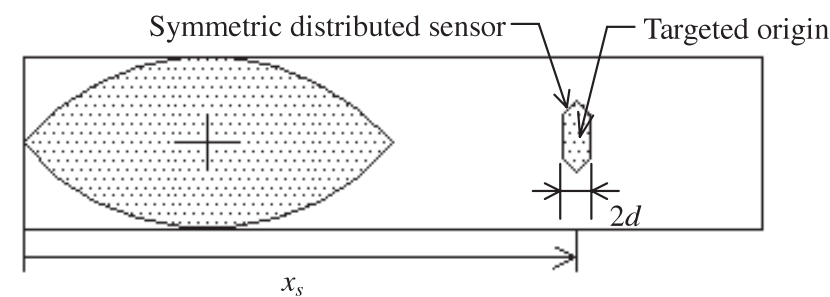

(a)
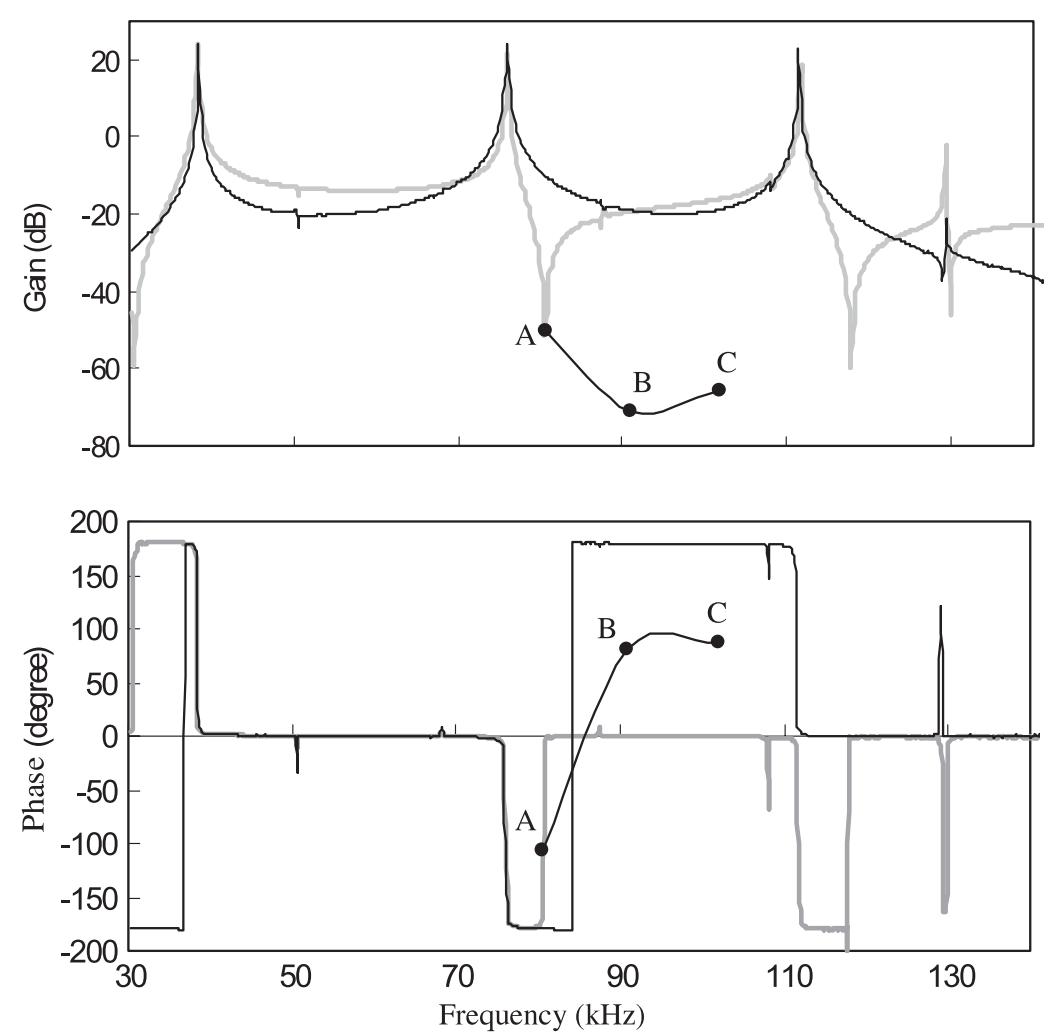

(b)

Figure 16. (a) Schematic of a symmetric distributed sensor. (b) Transfer function of a quasi-mode-two actuator and symmetric distributed sensor on a surface-to-surface piezoelectric plate, where the gray curve is the symmetric distributed sensor placed at $x_{\mathrm{s}}=(9 / 16) l$, and the dark curve is the one placed at $x_{\mathrm{s}}=(3 / 4) l$. The three black spots represent the zeros in the frequency response with the sensor placed at $x_{\mathrm{s}}=(9 / 16) l,(10 / 16) l,(11 / 16) l$ for point $\mathrm{A}, \mathrm{B}$, and $\mathrm{C}$, respectively.

that the point sensor on the lateral poling side of the adjoining piezoelectric plate is integrated along the thickness direction as derived in equation (26) and the integration path is as shown in figure 14(b). Thus the sign of mode 3 will change from negative to positive as the point sensor moves from a middle point to the boundary. That is, the sign from mode 1 to mode 3 is positive-negative-positive alternating for a point sensor placed near the boundary. The pole-zero alternating effect will not occur in this configuration. On the contrary, a zero will occur for a point sensor placed near the middle point of the piezoelectric plate. Figure 18 shows the experimental result with an identical distributed actuator and point sensor as shown in figure 16(a). We see that the zero can also be tailored by changing the location of the symmetric distributed sensor.

In summary, the pole-zero alternating phenomenon is a spatial relationship between the actuator and sensor as predicted by equation (15), and which can exist for any finite structure system under vibration. The transfer function between the actuator and the sensor mentioned above is of a different form from that of the case where identical sensors and actuators are placed at various locations. It should be noted that the spatial dependency between the sensor and the actuator pair determines the transfer function and this dependency cannot be examined by using the equivalent circuit model typically used to model piezoelectric materials.

Figure 19 is an example of utilizing the characteristics of a side-by-side piezoelectric plate with its first resonance frequency at $37.9 \mathrm{kHz}$ and its transfer function further modified by a second-order Butterworth low-pass filter with a corner frequency at $46.9 \mathrm{kHz}$. This designed transfer function offers the side-by-side piezoelectric plate a platform to develop an approximated single mode resonator operating at the first resonance mode of the longitudinal vibration (dark curve in figure 19). In this approach, the complexity of the selfresonance circuitry as in a piezoelectric transformer and piezoelectric gyroscope can be simplified since only one resonance mode appears in the transfer function of this kind of resonator. 

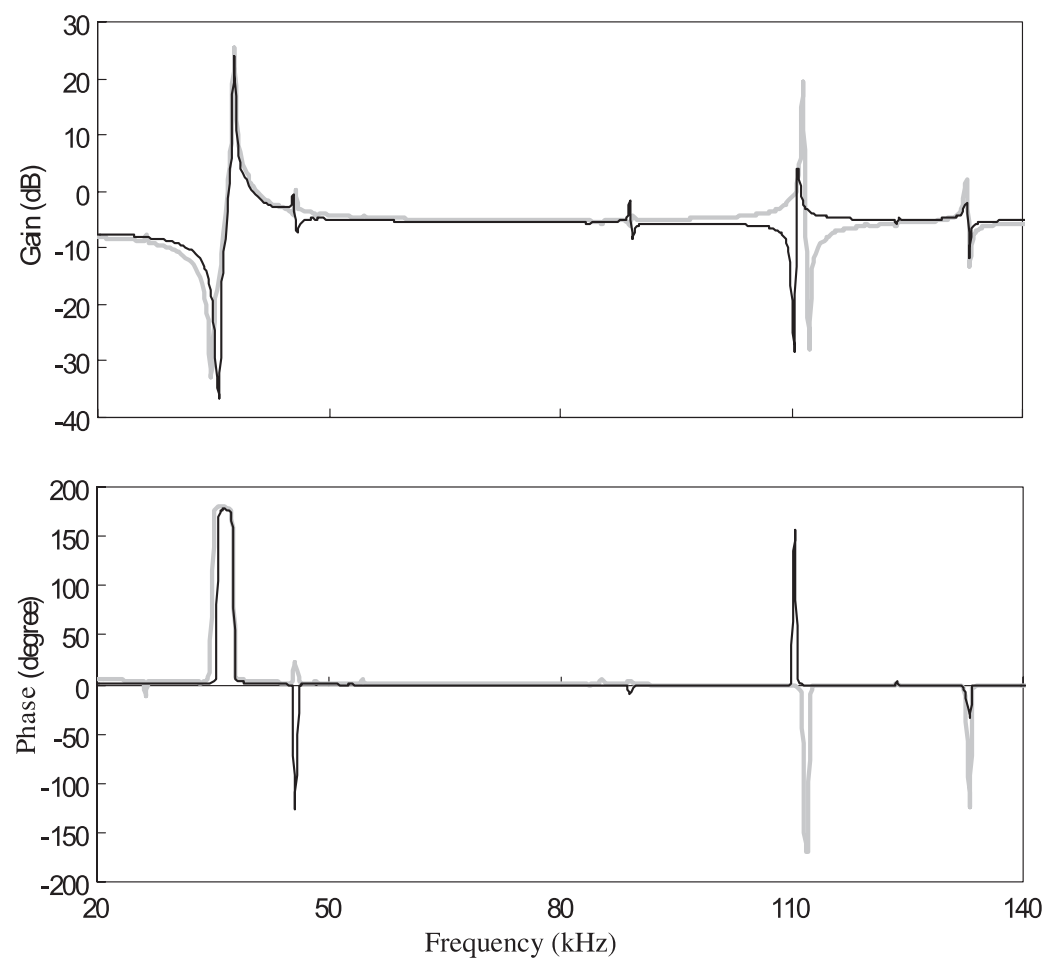

Figure 17. Transfer function of a uniform actuator and sensor on a side-by-side piezoelectric plate, where the dark curve and gray curves are the transfer functions for the full and $1 / 4$ length uniform sensors placed at the middle of the piezoelectric plate.
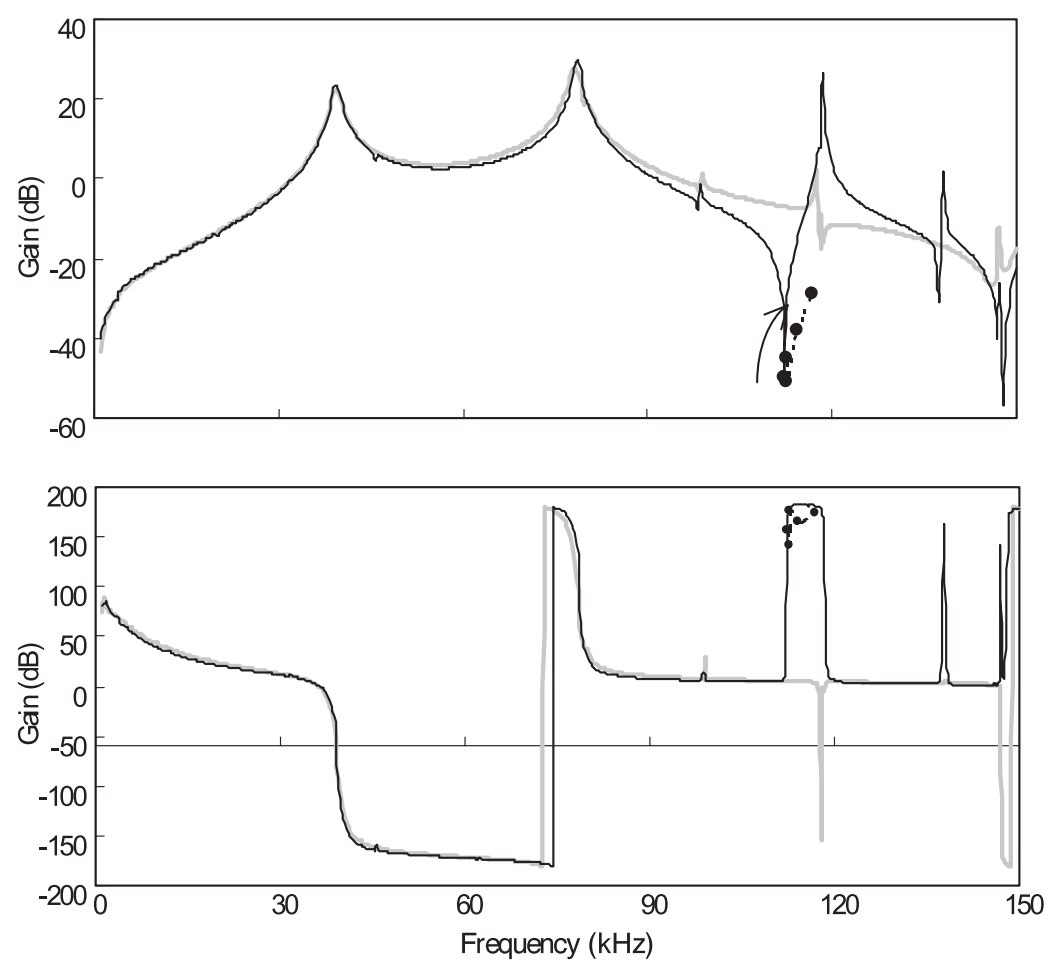

Figure 18. Transfer function of a mode-two actuator and symmetric distributed sensor on an adjoining piezoelectric plate, where the dark curve and gray curves are the transfer functions with symmetrically distributed sensors placed at $x_{\mathrm{s}}=(3 / 4) l$ and $(9 / 11) l$, and the black points are the zeros in the frequency response with the location of the symmetrically distributed sensor placed between $x_{\mathrm{s}}=(3 / 4) l$ and $(9 / 11) l$.

\section{Discussions}

It is clear from our outlined approach that electromechanical coupled systems are really a function of both spatial and temporal characteristics. Although it is quite common to treat electromechanical coupled systems by using an equivalent circuit concept, the underlying thought discussed in this paper emphasizes the importance of handling the design criteria in 

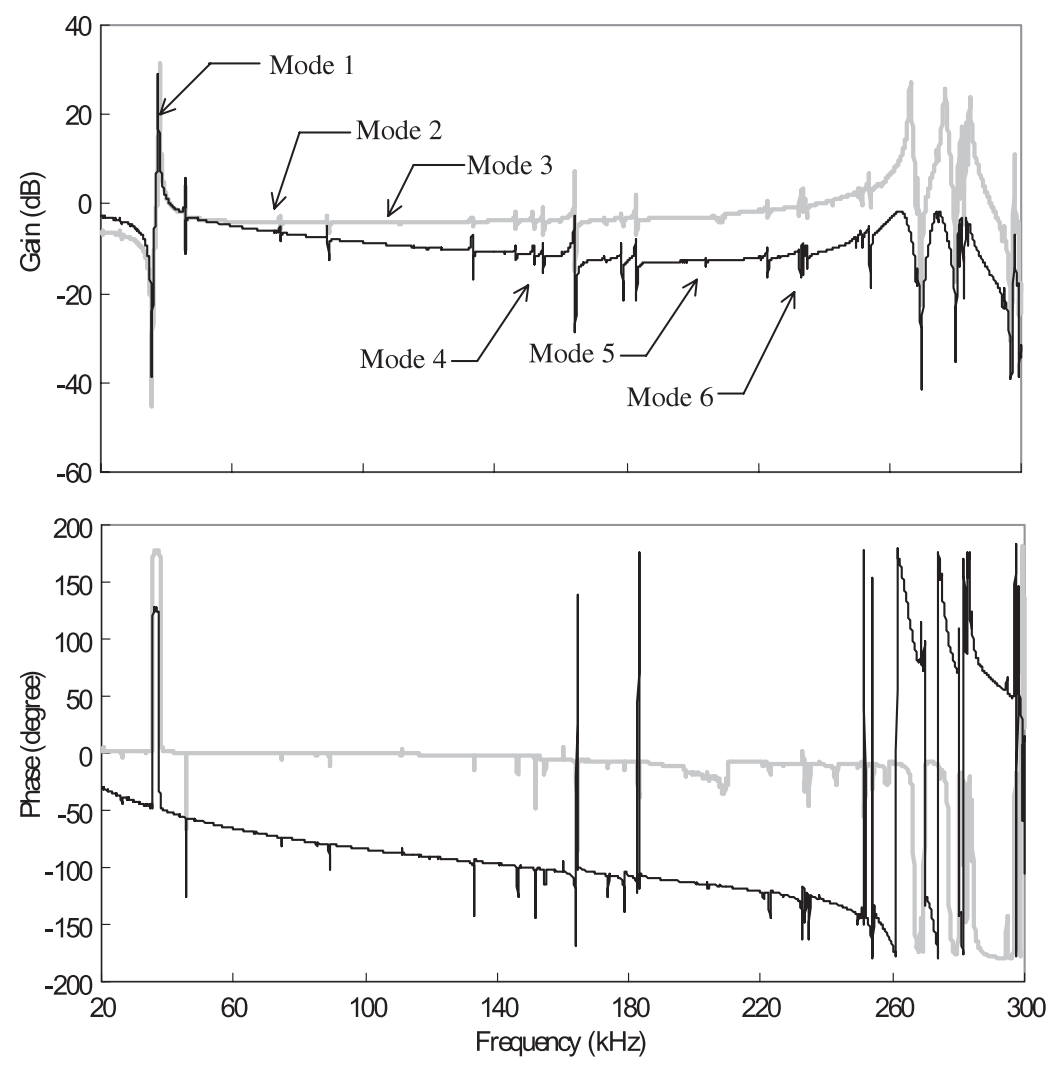

Figure 19. Transfer function of an approximated single mode resonator by a side-by-side piezoelectric plate (gray curve), and one with a second order low-pass filter used to filter out the contributions of the high order modes (dark curve).

both a temporal and spatial fashion. It should be noted that this concept is not limited to piezoelectric systems as applications such as capacitance sensors and piezoresistive sensors also possess identical characteristics. Thus, the APROPOS concept not only can be used to create the $P o D$ sensors as proved both theoretically and experimentally in this paper, but also can be used to extract information from a specific point on the specimen of interest. For example, even though strain gages have been developed for more than 100 years, strain gages that measure only a single point of a specimen are still unavailable. Today's strain gage measures the average strain of all areas located under the gage area. It is clear from equation (11) that the sensor output represents a transfer function gain tailoring with respect to the strain of the targeted origin. This aspect can thus also be used to retrieve strain at a single point, which in theory is infinitesimal and thus does not provide any meaningful information. More specifically, the APROPOS concept presented in this paper can be used to reveal the strain of an infinitesimal point of a mechanical system, which is of particular importance in the miniature devices found in today's micromachining processes.

The possibility of adopting an APROPOS device concept to develop a finite-area strain gage that can measure the strain of an infinitesimal point is very desirable. Traditionally, a Whetstone Bridge has been used to measure $\Delta R / R$ to monitor the strain $\varepsilon_{\mathrm{L}}$ [28], where $\Delta R$ and $R$ are the overall variation and total resistance of the piezoresistive material. However, the information only details a uniform distribution of strain, i.e., the strain distribution covered by the piezoelectric material is a constant. However, this concept does not apply if both of the above-mentioned assumptions do not hold up. Two situations show this particular problem. The first case is one where the strain gage is applied to study the dynamic response of a structure. Since there exist various resonance modes in a finite structure with a wide range of wavelengths, the information of low-wavelength resonance modes will be averaged out due to the larger sensing area. In this situation, the strain gage will then no longer be able to act as a point sensor and serve as a reliable sensor for dynamic testing. The second case is based on an application using a MEMS-based sensor design. As the micromachining structure ranges from millimeters to micrometers, the piezoresistive material that tries to follow the strain gage concept becomes difficult to implement as a smaller scale is required. Moreover, its sensitivity drops significantly since the length of the micromachined-based strain gage is not long enough. Thus a distributed piezoresistive lamina with the ability to measure the information of a local strain becomes a considerable issue, and adopting the concept of an APROPOS device serves as one of the possible ways to deal with this problem. Figure 20 shows a schematic of a pointdistributed strain gage designed with an APROPOS device. The fundamental design of a point-distributed strain gage can be easily drawn from figure 17 , noting that the cross-section of the targeted origin is the smallest. Since the value of the resistance is increased with a reduced cross-sectional area, the resistance at the targeted origin is the largest. Thus, the measured variation of the resistance will be enhanced at the targeted origin, and this is the reason why a point-distributed strain gage can serve as a point sensor with a distributed piezoresistive lamina. Thus the point-distributed strain gage 


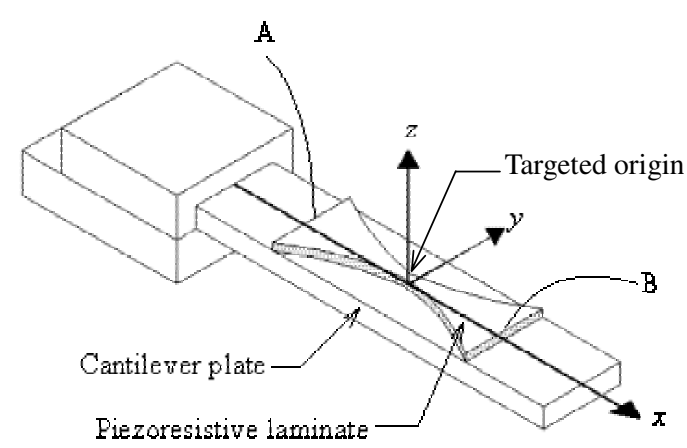

Figure 20. Schematic of a piezoresistive laminate designed using the concept of symmetric APROPOS devices.

serves as the only strain gage that can measure the strain information at a specific point and offers an appropriate sensing area for small structures such as MEMS systems.

\section{Conclusions}

Partial differential equations of space and time, instead of traditional equivalent circuit models developed based on ordinary differential equations, are found to be an essential element to introduce innovation into today's piezoelectric sensor and actuator systems. Dispersion relationships found in finite mechanical structures have been identified to provide us with the means to affect the transfer function in the frequency domain by pursuing spatial domain tailoring. That is, analyzing the mechanical vibration by a wavenumber and the spatial domain while studying the electrical performance from a frequency and time domain allows us to realize previously unfounded design freedom when compared to traditional electrical sensors and actuators. In summary, piezoelectric systems operate effectively only when all mechanical vibration, electrical characteristics, and interface circuits are all properly considered.

Combining the powerful design thinking offered by a method of image and window functions to design the weighting functions offered in a spatial domain, an APROPOS device concept was used to create point-distributed sensors which integrate the advantages of both point and distributed sensors. By taking into account evanescent waves and boundary effects, limitations faced by the spatial filter concept are thus eliminated. These point-distributed sensors have the advantage of ease-of-use similar to those of point sensors and in addition possess the design freedom offered by that of APROPOS devices, which provide a designer with an opportunity to adjust the system gain without affecting the system phase. That is, the classic dilemma of tailoring a system gain that will automatically lead to a loss of the phase margin can be circumvented. It was shown that all of these added advantages can lead to a much wider sensor bandwidth and to a much higher gain for a desired frequency range, which can also be equally applicable to traditional flexible structures. The concept of an APROPOS device applies not only to piezoelectric distributed sensors, but also to any other physical fields that can be used to offer spatial weighting to a finite structure. The concept of using an APROPOS device to implement a point-distributed sensor is one such example.
It serves as the only strain gage that can measure the specific strain information of a particular point and it can serve as a point sensor that possesses a sensing area that is large enough to provide good signal-to-noise ratio, even for small structures such as MEMS systems.

We studied the spatial relationships between actuators and sensors, full-field equations for surface-to-surface, side-byside, and adjoining piezoelectric plates vibrated longitudinally. It was verified theoretically and experimentally that the polezero alternating phenomenon really is a spatial effect between a sensor and actuator as the transfer function represents the fully coupled mechanical characteristics of the actuator and sensor pairs. We know that there will exist a zero between the two structure resonance modes, if and only if the signs of these modes are identical. We show that the sign of the structure resonance mode is determined by the spatially distributed actuator and sensor. As for the point actuator and sensor, the sign is determined by its location with respect to the modal strain of the structure. On the other hand, the sign of the distributed actuator and sensor is determined by the results of the surface integration with respect to the monitored strain distribution. We conclude that the pole-zero alternating phenomenon can tailor the transfer function of a finite structure driven and sensed by an actuator and a sensor. An approximated single mode resonator using a side-by-side piezoelectric plate was also proved possible. It should be noted that the pole-zero alternating phenomenon is a spatial property, which cannot be molded by a simple equivalent circuit.

\section{Acknowledgments}

The authors would like to acknowledge the continuous and generous financial support of this research from the National Science Council of Taiwan, Republic of China through Grant NSC-92-2622-E-002-006, and from the Ministry of Economic Affairs, Republic of China, through Grant \# 92-EC-17-A-05S1-0017. Appreciation also goes to Measurement Specialties, Sensor Products Division (MSI) for continuously providing us with all the $\mathrm{PVF}_{2}$ films used in fabricating the piezoelectric devices discussed in the research work. The financial and material support of AdvanceWave Technology, Inc. is also greatly appreciated as well. Also, thanks goes to ELECERAM Technology Co. Ltd for continuously providing us with the piezoelectric transformers used in this research work.

\section{References}

[1] Cady W G 1964 Piezoelectricity vol 1 (New York: McGraw-Hill) pp 1-8

[2] Bode H W 1945 Network Analysis and Feedback Amplifier Design (New York: Van Nostrand-Reinhold)

[3] Hsu Y H and Lee C K 2002 Targeted origin placement for the autonomous gain-phase tailoring of piezoelectric sensors Smart Mater. Struct. 11 444-58

[4] Hsu Y H and Lee C K 2003 Extending bandwidth of rotational accelerometers by autonomous gain-phase tailoring Smart Mater. Struct. 12 210-6

[5] Das A and Wada B 2001 Selected Papers on Smart Structures for Spacecraft (Bellingham, WA: SPIE Optical Engineering Press)

[6] Serridge M and Licht T R 1986 Piezoelectric Accelerometers and Vibration Preamplifiers (Denmark: Brüel \& Kjær) 
[7] Bailey T and Hubbard J E 1985 Distributed piezoelectric-polymer active vibration control of a cantilever beam J. Guid. Control Dynam. 8 605-11

[8] Lee C K and Moon F C 1990 Modal sensors/actuators ASME J. Appl. Mech. 57 434-41

[9] Collins S A, Miller D W and Von Flotow A H 1994 Distributed sensors as spatial filters in active structural control J. Sound. Vibration 173 471-501

[10] Micromachined Servo Accelerometer-Endevco Model MSA100, Intertechnology Inc., 1 Scarsdale Road. Don Mills, ON M3B 2R2 2003 http://www.intertechnology.com/Endevco/

[11] Hsu Y H and Lee C K 2001 Miniature free-fall sensors J. Intell. Mater. Syst. Struct. 12 223-8

[12] Martin G D 1978 On the control of flexible mechanical systems PhD Dissertation Stanford, California, USA

[13] Madou M 1997 Fundamentals of Microfabrication (New York: CRC Press)

[14] Bracewell R N 1978 The Fourier Transform and Its Application 2nd edn (New York: McGraw-Hill)

[15] Harris F J 1978 On the use of windows for harmonic analysis with the discrete Fourier transform Proc. IEEE 66 51-83

[16] Graff K F 1975 Wave Motion in Elastic Solids (New York: Dover)

[17] Lee C K 1987 Piezoelectric laminates for torsion and bending modal control: theory and experiment $P h D$ Dissertation Institute of Applied Mechanics, Cornell University
[18] Kawai H 1969 The piezoelectricity of poly (vinylidene fluoride) Japan. J. Appl. Phys. 8 975-6

[19] Ottesen M 1987 Apparatus for detecting and correcting extensive vibration in a disk file IBM Tech. Discl. Bull. 30 81-2

[20] Lee C K, Munce A C Jr and O’Sullivan T C 1996 Disk drive with acceleration rate sensing US Patent Specification $5,521,772$

[21] Hsu Y H and Lee C K 2001 Miniature free-fall sensors J. Intell. Mater. Syst. Struct. 12 223-8

[22] Meirovitch L 1986 Element of Vibration (New York: McGraw-Hill)

[23] Berlincourt D A and Sliker L S 1973 Piezoelectric transformer US Patent Specification 3736446

[24] Kudo S, Konno M, Sugawara S and Yoshida T 1990 Consideration on equivalent mechanical circuits for vibratory gyroscope IEEE Ultrasonics Symp. pp 397-400

[25] Tiersten H F 1969 Linear Piezoelectric Plate Vibrations (New York: Plenum)

[26] Katz H W 1959 Solid State Magnetic and Dielectric Devices (New York: Wiley) pp 94-126

[27] Hsu Y H, Lee C K and Hsiao W H 2003 Optimizing piezoelectric transformer for maximum power transfer Smart Mater. Struct. 12 373-83

[28] Horowitz H and Hill W 1995 The Art of Electronics (New York: Cambridge University Press) 